\title{
Effect of mechanical resonance on Josephson dynamics
}

\author{
C. Padurariu, C. J. H. Keijzers, and Yu. V. Nazarov \\ Kavli Institute of NanoScience, Delft University of Technology, Lorentzweg 1, 2628 CJ, Delft, The Netherlands
}

(Received 26 December 2011; published 25 October 2012)

\begin{abstract}
We study theoretically dynamics in a Josephson junction coupled to a mechanical resonator looking at the signatures of the resonance in dc electrical response of the junction. Such a system can be realized experimentally as a suspended ultraclean carbon nanotube brought in contact with two superconducting leads. A nearby gate electrode can be used to tune the junction parameters and to excite mechanical motion. We augment theoretical estimations with the values of setup parameters measured in one of the samples fabricated. We show that charging effects in the junction give rise to a mechanical force that depends on the superconducting phase difference and can excite the resonant mode. We develop a model that encompasses the coupling of electrical and mechanical dynamics. We compute the mechanical response (the effect of mechanical motion) in the regime of phase and dc voltage bias. We thoroughly investigate the regime of combined ac and dc bias where Shapiro steps are developed and reveal several distinct regimes characteristic for this effect. Our results can be immediately applied in the context of experimental detection of the mechanical motion in realistic superconducting nanomechanical devices.
\end{abstract}

DOI: 10.1103/PhysRevB.86.155448

PACS number(s): 85.85.+j, 74.45.+c, 73.23.Hk

\section{INTRODUCTION}

Nanoscale electromechanical systems (NEMS) convert small amplitude mechanical motion into measurable electrical currents. ${ }^{1}$ Devices based on NEMS have found applications as sensitive detectors of mass, ${ }^{2}$ force, ${ }^{3}$ and electrical charge. ${ }^{4}$ Considerable research efforts have been dedicated to improving detection sensitivity by fabricating devices with higher resonance frequencies, lower damping rates (high quality factors), and larger coupling between electrical and mechanical degrees of freedom.

The problem of detecting the quantum state of a macroscopic mechanical resonator gave rise to several measuring schemes, proposed $^{5,6}$ as well as realized. ${ }^{7}$ Improvements in device fabrication have pushed the sensitivity threshold to the quantum limit. ${ }^{7}$ In addition, new techniques for cooling mechanical motion have been proposed. ${ }^{8,9}$ The use of superconducting devices, in particular, superconducting qubits to detect and control the mechanical motion is in focus of modern research. ${ }^{7,10}$ It gives rise to a growing interest in techniques of coupling NEMS to superconducting circuits.

Superconducting nanodevices frequently use Coulomb blockade that makes their transport properties sensitive to the gate voltages. The same gate voltage can be used to excite the mechanical motion that is detected from the change of dc transport properties of the device. ${ }^{13-15}$ Without superconductors, this scheme has been successfully realized for a metallic single-electron transistor ${ }^{17}$ and for a Coulombblockaded quantum dot in an ultra-clean carbon nanotube (CNT). ${ }^{15}$ The results revealed high resonance frequencies, reaching gigahertzs, and unprecedented quality factors of the order of $10^{5}$. These devices can be made superconducting by connecting them to superconducting leads and providing sufficiently large coupling between the states of the lead and device. We have successfully realized Josephson junctions based on ultraclean CNTs. The supercurrent observed demonstrates a pronounced gate-voltage sensitivity that indicates a well-developed Coulomb blockade. ${ }^{27}$

A very interesting proposal that combines Josephson dynamics and mechanical resonator has been recently put forward by Gothenburg collaboration. ${ }^{21}$ The authors consider an ideal ballistic CNT between two superconducting leads biased at voltage $V$. Owing to Josephson relation, the current in the nanotube oscillates at frequency $\omega_{j}=2 \mathrm{eV} / \hbar$. The authors notice that in external magnetic field this gives rise to an oscillating Lorentz force. If the frequency matches the frequency of the mechanical resonator, the force excites mechanical motion which rectifies the Josephson current enabling the observation of the effect in dc electric response of the junction. One would observe a narrow current peak in $I-V$ characteristics of the device. The same mechanism is responsible for Fiske steps: ${ }^{25}$ the difference is that in Fiske experiments the resonance is electrical rather than mechanical.

This provides us a motivation for the present theoretical study. We address superconducting NEMS where a mechanical resonator is integrated with a superconducting circuit element, a Josephson junction. The details of the setup are given in Sec. II. In this work, we (i) explore the coupled dynamics of the oscillator displacement and superconducting phase difference and (ii) describe the manifestations of mechanical motion in superconducting current under various bias conditions. The goal is to list experimentally observable effects. We investigate in particular detail the effect of mechanical motion in the context of Shapiro steps as the most promising one with respect to experimental detection.

Part of our results are valid for any device combining Josephson effect with mechanical resonance. However, we mostly concentrate on a class of particularly successful NEMS devices: suspended metallic CNT connected to superconducting leads. We have fabricated and studied such devices. Since our research is theoretical we strive for generality presenting thereby analytical and numerical estimations and demonstrating reasonable values and ranges of the parameters in use. Unfortunately, the mere choice of parameters to be in the reasonable range does not immediately quantify the values of the results obtained: they may vary significantly and there is no simple scaling present. The same is true for the experiments: the devices produced vary in length, in stress as indicated by measured frequencies, and in the conductance 
that estimates the Josephson coupling. In this situation, we supplement general estimations with calculations for a set of concrete numerical values of the parameters in use. This set is given in Sec. IIIF. This parameter choice is therefore rather arbitrary, although it matches closely one of the devices made.

Studies of CNT Josephson junctions have shown that their Josephson energy can be modulated by the gate-induced charge on the CNT. Under these circumstances, the electrostatic energy of the system requires a special consideration (see Sec. III A). With this, we formulate the notion of Josephson mechanicalforce (see Sec. III B). This is one of the main results of our work since we reveal a mechanism of phase-dependent mechanical driving which is different and generally more important than that considered in Ref. 21. General equations of motion including this force (see Sec. IIID) need to be further analyzed to reveal which of the three competing nonlinearities is the most relevant one. The result of rather involved analysis performed in Sec. III D shows that the most important nonlinearity is the intrinsic mechanical nonlinearity, at least, for CNT-based superconducting mechanical resonators.

In fact, the mechanical nonlinearity has been ignored in Ref. 21. In their case, the resonant enhancement of oscillating amplitude can only be stabilized by the feedback from nonlinear Josephson dynamics. This brought the authors of Ref. 21 to the analysis of strong feedback between the displacement and superconducting phase. Our results thus show that such feedback, although interesting, can not be realized in practical CNT devices since it is the mechanical nonlinearity that stabilizes the resonant growth of the oscillation amplitude.

Neglecting phase and charge nonlinearities in comparison with the mechanical one permitted us to simplify the equations drastically. Final equations and the workflow to determine the quantity of interest-the mechanical response current $I_{m}-$ are given in Sec. IIIE. Throughout the Sec. III we provide detailed estimations of the displacement, force and electrical current scales involved. It is our conclusion that the mechanical response in our devices should be small. For the parameter choice made, the mechanical response is at the scale of $10^{-3}$ of the critical current. ${ }^{22}$ Further on, we apply the expressions obtained for a variety of bias conditions.

In Sec. IV, we discuss the phase bias. We predict the phasedependent shift of the mechanical resonance frequency that is an important signature of coupled dynamics. Our estimation of the frequency shift shows that it can be easily observable, being significantly larger than the resonance width. In addition, we show that a dc mechanical response current develops upon mechanical excitation of the device. We elaborate on the line shape of the mechanical response current showing that for weak driving conditions, it is an usual Lorentzian dependence, while, in contrast, for strong driving conditions that induce a nonlinear mechanical response, the line shape becomes asymmetric, acquiring a Fano-type shape (see Sec. IV A).

Section V is devoted to dc voltage bias conditions. Resonant mechanical driving occurs at Josephson frequency matching the resonance frequency of the mechanical resonator, or an integer fraction of this frequency by higher harmonics (see Sec. V A). We study the resulting Fiske-type mechanical response and give the estimations of the effect. For completeness, we also shortly discuss the possibility of parametric excitation. We show by estimation that the emergence of parametric mechanical response requires a large Josephson energy, at least, an order of magnitude larger than that achievable in practical devices (see Sec. V A).

Sections VI and VII are devoted to the dynamics in the presence of external ac drive, in the regime where the Josephson junction gives rise to well-developed Shapiro steps. ${ }^{26}$ One of the motivations of the use of Shapiro steps is the better synchronization conditions in comparison with dc voltage bias. This can be seen as follows. The big quality factor $Q$ of the nanomechanical resonance results in a narrow Fiske-type current peak (discussed in Sec. V). Its width in voltage can be estimated as $\delta V \simeq V / Q$. The observation of such a narrow feature imposes a severe limitation on voltage noise $S_{V}$ : to resolve the peak one must achieve $S_{V} \ll(e / \hbar) V / Q$. This may be challenging under realistic experimental circumstances. There is a way out: the voltage can be synchronized with the frequency of external irradiation. This happens at Shapiro steps and effectively reduces the voltage noise.

In Sec. VI, we study the mechanical response at the Shapiro steps in the regime where the ac driving frequency matches the resonant frequency, and present the peculiarities of this response. We derive explicit analytical expressions of the mechanical response as a function of ac driving amplitude and illustrate them with plots. We concentrate on the effect seen in dc current measurement, that is, the modification of the width and position of Shapiro steps. We show that the response at the first Shapiro step (see Sec. VI A), which develops at the position of the Fiske-type current peak, is qualitatively different from that at the higher Shapiro steps (see Sec. VI B). In Sec. VII, we present the same considerations for the case of the nonresonant driving that in the regime of Shapiro steps can efficiently excite the mechanical motion owing to Josephson nonlinearities.

Our preliminary experimental results show corresponding features. They will be presented elsewhere ${ }^{27}$ upon completion of detailed analysis and comparison with our theoretical findings. Our concluding remarks are presented in Sec. VIII.

\section{THE SETUP}

The setup under consideration is sketched in Fig. 1 where we concentrate on a case where both the Josephson junction

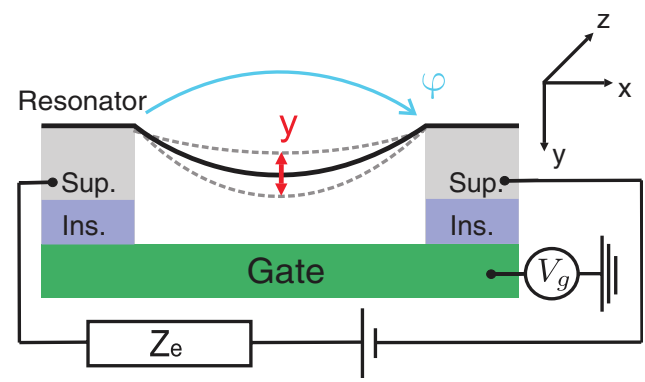

FIG. 1. (Color online) The setup. The sketch presents the mechanical resonator realized as a CNT suspended over two superconducting leads isolated from the back gate electrode. The CNT center is displaced in the $y$ direction by an electrostatic force produced by the gate voltage. The superconducting leads are parts of an electrical circuit characterized by an impedance $Z_{e}$. The setup can be biased by either voltage or current source. 
and the mechanical resonator are realized with the same single CNT. Even in this case, the coupling between mechanical and electrical degrees of freedom is relatively weak. This allows us to describe the electrical and mechanical aspects of the setup separately. We provide the description in this section, while in the next section we concentrate on the coupling.

\section{A. Electrical setup}

We consider a conducting link between two superconducting leads (the CNT in Fig. 1). In general, the current flowing in this junction is a complicated nonlinear and time-delayed function of superconducting phase difference between the leads. However, we assume that in the relevant frequency range the current response of the junction is superconducting and instant.

The junction is included into an external electric circuit and the voltage drop at the junction is related to the time derivative of Josephson phase, $\dot{\varphi}=2 e V / \hbar$. In general, a circuit that connects the leads can be described by a complex frequencydependent impedance $Z_{e}(\omega)$ in series with a voltage source $V_{b}$. We typically assume that $Z_{e}$ by far exceeds the typical junction impedance at low frequencies while at frequency scale of Josephson generation frequency $\omega \simeq e V / \hbar, Z_{e}(\omega)$ is negligible in comparison with the junction response. In this case, the junction is current biased at low frequencies with $I_{b}=$ $V_{b} / Z_{e}(0)$ and voltage biased at Josephson frequencies. While this scheme looks different from the traditional resistively shunted junction model (RSJ) where the external impedance is connected in parallel and the junction is current biased, it is equivalent to a generalized RSJ upon transforming the impedance and the voltage source. For instance, the linear part of possible quasiparticle response of the junction can be incorporated into $Z_{e}(\omega)$.

In addition, the junction is affected by the gate electrode biased by voltage source $V_{g}$. The bias and gate circuits are disconnected at zero frequency. At finite frequency, there is a cross talk between the circuits, which is difficult to eliminate or even characterize in realistic experimental circumstances. We account for that by correlating ac parts of the voltage sources $V_{b, g}$. For instance, if the gate voltage consists of a dc part and a harmonic signal at frequency $\Omega, V_{g}(t)=$ $V_{g 0}+\tilde{V}_{g} \cos (\Omega t+\chi)$, the bias voltage source should also oscillate at the same frequency, $V_{b}(t)=V_{b 0}+\tilde{V}_{b} \cos (\Omega t)$. The ratio of two ac amplitudes and their mutual phase shift $\chi$ is determined by details of the crosstalk. We will show below that the interference of these two ac signals may strongly affect the dc currents in the junction.

The superconducting current is determined by the instant phase difference, $I(t)=I[\varphi(t)]$. In this case, it is related to the Josephson energy $E_{j}$ of the junction,

$$
I=(2 e / \hbar) \partial E_{j}(\varphi) / \partial \varphi .
$$

It is essential for us that the Josephson energy is not only a function of phase difference but also depends on the gate voltage through the charge $q=C_{g} V_{g}$ induced in the resonator, $E_{j}=E_{j}(q, \varphi)$.

For a nanotube device, the origin of this charge sensitivity is (weak) Coulomb blockade of electrons in the middle of the nanotube. The nanotube can be viewed as two junctions in series, those being formed at contact with metallic leads. If the conductance of the junctions is smaller or comparable with the conductance quantum $G_{Q} \equiv e^{2} /(\pi \hbar) \approx 7.75 \times 10^{-5} \Omega^{-1}$ Coulomb interactions become important and set a quasiperiodic dependence of Josephson energy on $q$ with a period $2 e$. This corresponds to charge quantization in the middle of the nanotube. We routinely observe the quasiperiodic modulation of superconducting currents in fabricated nanotube devices. The modulation can be tuned by changing the gate voltage at scale of $q \simeq 10-100 e$ from values of the order of one to several percent. Big modulation and well-developed Coulomb blockade require big junction resistances, this strongly suppresses the superconducting current. It is therefore advantageous to have intermediate resistances $R \simeq G_{Q}^{-1}$. At $R=5 \mathrm{k} \Omega$, we typically observe $30 \%$ modulation.

The superconducting current is a periodic function of the phase $I(\varphi)=I(\varphi+2 \pi)$ and therefore can be expanded in harmonics as ${ }^{23}$

$$
I(q, \varphi)=I_{1}(q) \sin (\varphi)+\sum_{n=2}^{\infty} I_{n}(q) \sin (n \varphi),
$$

If one neglects all harmonics except the first one, $I_{1}$ gives the critical current of the Josephson junction. We will typically assume this, and will mention the effect of higher harmonics only if it is crucial.

\section{B. Mechanical setup}

Mechanical resonators can be realized in a variety of ways. ${ }^{11}$ In many cases, the adequate description of the resonator can be achieved with a minimum model that accounts for excitations of a single resonator mode, neglecting coupling to any other modes. The minimum model is given by the following equation of motion for a displacement variable $y$ :

$$
\ddot{y}+\Gamma \dot{y}+\omega_{0}^{2} y-\alpha y^{2}-\beta y^{3}=F(t) / M .
$$

Here, $F(t)$ is the time-dependent driving force, $M$ is the effective mass corresponding to the mode, $\omega_{0}$ stands for the resonant frequency, $\Gamma \ll \omega_{0}$ is the damping rate, and $\beta$ is the parameter describing the leading cubic nonlinearity. ${ }^{12}$ The cubic nonlinearity provides the important restriction on the magnitude of the displacement at resonant frequency as a reaction on resonant force. We also keep the second-order nonlinearity $\alpha$. Although it is not important in analysis of the reaction at resonant force, it describes the shift of the resonant frequency due to constant force.

Our preferable realization of mechanical resonator is a suspended ultraclean $\mathrm{CNT}^{13-15}$ that demonstrates best quality factors observed so $\operatorname{far}\left(Q \equiv \omega_{0} / \Gamma \simeq 10^{5}\right)$. In this section, we review the parameters of the minimal model for this realization. In the setup shown in Fig. 1, the nanotube displacement from equilibrium position and the driving force are in the $y$ direction towards the gate, that is, perpendicular to the nanotube axis. The mechanical variable $y(t)$ is the displacement of the midpoint of the nanotube.

In the case of a CNT, the adequate model of mechanical properties involves a suspended thin cylindrical rod clamped at both ends where the nanotube touches the metal leads. The parameters are the rod length $L$, the cylinder radius $r$, and the tube cross section area $S$. In our experiments, 
$L \simeq 0.3-0.5 \mu \mathrm{m}, r \simeq 1-2 \mathrm{~nm}$, and $S \simeq 2 \pi r a \simeq 2.1-4.3 \mathrm{~nm}^{2}$ for a single-wall nanotube, $a \simeq 0.34 \mathrm{~nm}$ being the layer spacing in graphite. The relevant elastomechanical material constants, carbon Young's modulus $E$ and graphite density $\rho$ are estimated as $E \simeq 10^{12} \mathrm{~J} / \mathrm{m}^{316}$ and $\rho \simeq 2.2 \mathrm{~g} / \mathrm{cm}^{3}$. The bending modes of the rod and their complete dynamics are described by the Euler-Bernoulli equation of motion. ${ }^{19,20}$

We concentrate on the lowest-frequency bending mode that has no nodes in the rod and therefore is easy to excite. The resonant frequency can be tuned by "tightening" the tube, that is, changing the elastic tension. This is achieved by applying a sufficiently big dc gate voltage $V_{g 0}$. The resulting electrostatic force strives to elongate the nanotube, thus producing the tension. In such a way, the resonant frequency can be increased by a factor of three in comparison with that of the "loose" nanotube. For estimations, we concentrate on the case of loose rod. In this case, the resonance frequency corresponding to the lowest CNT bending mode can be estimated in terms of the bending spring constant and the carbon mass density $\omega_{0} \simeq$ $22.4 \sqrt{E I / \rho S} L^{-2}$, where $I \simeq S r^{2} / 2$ is the moment of inertia ${ }^{18}$ of the CNT cross section $I=\int x^{2} d S$, as introduced in Ref. 19 . In our devices of length $L=0.3-0.5 \mu \mathrm{m}$, the frequency is $\omega_{0} / 2 \pi \simeq 0.30 . .0 .84 \mathrm{GHz}$, similar to frequencies reported in Ref. 15. The effective force is evaluated using the eigenfunction of the mode $\xi(x) \equiv y(x, t) / y(t), F=\int_{0}^{L} d x f(x) \xi(x)$, $f(x)$ being the force per unit length. For electrostatic forces, an ad hoc assumption is that the force distribution is uniform, so the total force is $F_{t}=f L$. In this case, $F \simeq$ $0.53 F_{t}$. The effective mass is given by $M=\rho S \int_{0}^{L} d x \xi^{2}(x)$, $M \simeq 0.41 \rho S L \simeq 4.1-6.8 \times 10^{-22} \mathrm{~kg}$. The cubic nonlinearity originates from the tension produced by the nanotube displacement, the corresponding parameter can be estimated as $\beta \simeq 40 \mathrm{ES} / M L^{3} \simeq \omega_{0}^{2} / r^{2} \simeq 1.8-5.5 \mathrm{GHz}^{2} \mathrm{~nm}^{-2}$, assuming uniform distribution of force along the length of the rod. The second-order nonlinearity $\alpha$ vanishes for loose straight rod for symmetry reasons. However, it becomes significant if the rod is tightened such that its frequency change with respect to the loose rod value $\omega_{0}$ is of the order of $\omega_{0}$. In this case, the nonlinearity is obtained as $\alpha=3 \beta y_{0}, y_{0}$ being the equilibrium displacement induced by the tightening.

If $F(t)$ oscillates at frequency $\omega$ close to the resonant frequency, Eq. (3) can be solved in resonant approximation for the complex amplitude $\tilde{y}$ :

$$
\tilde{y}=\frac{\tilde{F}}{2 M \omega_{0}} \frac{-1}{v+i \Gamma / 2+\left(\beta^{\prime} / 2 \omega_{0}\right)|\tilde{y}|^{2}},
$$

with $\tilde{F}$ being the complex force amplitude. Here, we introduce the detuning $v \equiv \omega-\omega_{0}$ implying that $|v| \lesssim \omega_{0}$. We also introduce the Duffing parameter $\beta^{\prime}=\beta+\alpha^{2} / \omega_{0}^{2} \simeq \omega_{0}^{2} / r^{2}$ corresponding to the amplitude-dependent frequency shift. In our experiments, we estimate $\beta^{\prime} \simeq 3.6-11 \mathrm{GHz}^{2} \mathrm{~nm}^{-2}$.

We will rewrite Eq. (4) in dimensionless form introducing a critical amplitude $y_{c}, y_{c}=\sqrt{\omega_{0} \Gamma / \beta^{\prime}}$. At this amplitude scale, the response of the resonator becomes a two-valued function of detuning (see Fig. 2). For a CNT, it can be estimated as $y_{c}^{2} \simeq r^{2} / Q$, which corresponds for our experiments to $y_{c} \simeq 3.2-6.4 \mathrm{pm}$. The driving force corresponding to $y_{c}$ is $F_{c}=M \beta^{\prime} y_{c}^{3}=M \omega_{0}^{2} y_{c} / Q$. We estimate it for a CNT $F_{c} \simeq$ $10^{2} E S(r / L)^{3} Q^{-3 / 2} \simeq(1.2-2.4) \times 10^{-18} \mathrm{~N}$.
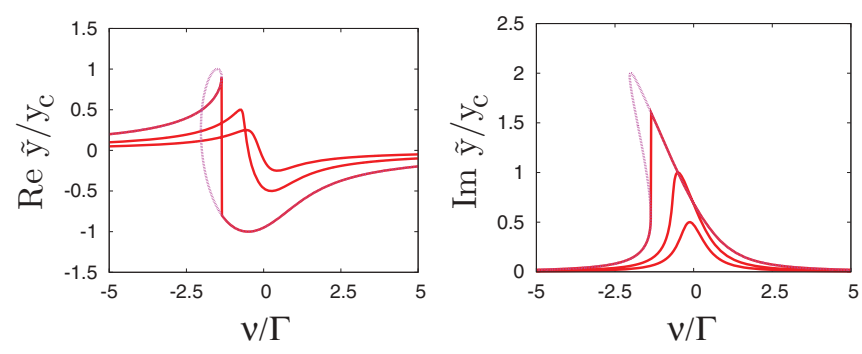

FIG. 2. (Color online) (Left) Real part of the complex displacement amplitude Re $\tilde{y}$ vs detuning. (Right) Imaginary part of the complex displacement amplitude Im $\tilde{y}$ vs detuning. The three curves in each panel correspond to $|\tilde{F}| / F_{c}=0.5,1,2$.

The dimensionless form of Eq. (4) is

$$
\frac{\tilde{y}}{y_{c}}=\frac{\tilde{F}}{F_{c}} R\left(\frac{v}{\Gamma}, \frac{\tilde{F}}{F_{c}}\right) ; \quad R(a, b)=\frac{-1}{2 a+|b|^{2}|R(a, b)|^{2}+i} .
$$

Here, we have introduced a dimensionless complex response function $R(v, \tilde{F})$. In the linear regime $|\tilde{F}| \ll F_{c}$, its dependence on $\tilde{F}$ can be neglected: $R(a, b)=(2 a+i)^{-1}$.

Figure 2 shows the real and imaginary parts of $\tilde{y}$ as a function of detuning for three values of the driving force amplitude that correspond to quasilinear, critical, and bistable regimes.

\section{COUPLING AND NONLINEARITIES}

In this section, we analyze the coupling between mechanical and electrical degrees of freedom. The coupling manifests as two quantities: displacement-dependent current and phasedependent mechanical force. Both quantities emerge from electrostatic effects, therefore we will start with a detailed discussion of electrostatic energy in the setup, finding the induced charge for a given mechanical displacement and phase. We then use the induced charge to express the forces and superconducting current. By doing so, we assume that the typical time of charge equilibration is much shorter than the typical time scale $\omega_{0}^{-1}$ of the mechanical motion. We compare the electrostatic phase-dependent force with Lorentz force proposed in Ref. 21. We derive the coupled equations of motion governing the Josephson and mechanical dynamics and identify the dominant source of nonlinear behavior.

\section{A. Electrostatic energy}

The junctions connecting the middle of the nanotube to the leads have intermediate resistance, so that the middle of the nanotube forms a Coulomb island that is neither isolated from, nor ideally connected to the leads. While the case of good isolation ${ }^{20}$ can be easily treated microscopically, the situation of intermediate conductances is difficult to quantify from a microscopic calculation. However, the situation can be completely analyzed at the phenomenological level. At this level, the analysis is a case of elementary nonlinear electrostatics. In comparison with Ref. 20, the analysis adds some important and less obvious details, so we choose to outline it at a comprehensive level. 
To start with, let us assume that the capacitance to the gate is vanishingly small while $V_{g}$ is diverging such that the charge induced to the Coulomb island by the gate $q=C_{g} V_{g}$ tends to a constant limit. A part of the ground-state energy of the setup $E_{c}(q)$ depends on $q$. General Coulomb-blockade considerations $^{24}$ imply that this part is a (quasi) periodic function of $q$ with a period of $2 e$. In the limit of full isolation, for instance, this energy is piecewise parabolic, $E_{c}(q)=E_{C} \min _{N}(N-q / 2 e)^{2}, N$ being an integer number of extra Cooper pairs stored in the island. In general, it is a smooth function of $q$ and may depend on the superconducting phase difference $\varphi$ and, in principle, on mechanical displacement $y$. This energy results in a nonzero electrostatic potential difference between the island and the leads, $V(q)=\partial E_{c}(q) / \partial q$.

Let us now turn to finite $C_{g}$ and therefore finite $V_{g}$ that is the potential difference between the leads and the gate electrode. Since this is not the potential difference between the island and the gate anymore, the induced charge $q$ is not equal to $C_{g} V_{g}$. Rather, it is determined from the voltage division between two capacitors: $C_{g}$ and one between the island and the leads. The total voltage difference $V_{g}$ is the sum of the voltage drops at the two capacitors,

$$
V_{g}=\frac{q}{C_{g}}+V(q)
$$

The charge is then found from this equation that can be rewritten as

$$
q=C_{g} V_{g}-C_{g} \frac{\partial E_{c}(q)}{\partial q} .
$$

We note that this is equivalent to the minimization of the total electrostatic energy with respect to $q$,

$$
E=\min _{q}\left(E_{c}(q)+\frac{q^{2}}{2 C_{g}}-q V_{g}\right) .
$$

Indeed, the condition of the energy minimum coincides with Eq. (6).

There are two implicit dependencies in this equation that distinguish it from pure electrostatics, and that we make explicit now. First of all, the electrostatic energy depends on the mechanical displacement of the nanotube. Geometric considerations suggest that this dependence can be ascribed to $C_{g}$ : indeed, the modification of capacitance to the gate is linear in $y, C_{g} \rightarrow C_{g}+\left(d C_{g} / d y\right) y$, while the modification of $E_{c}$ is expected to be $\propto y^{2}$. Secondly, the electrostatic energy depends on the superconducting phase difference: indeed, the Josephson energy is just the phase-dependent part of $E_{c}, E_{c}(q, \varphi)=\bar{E}_{c}(q)+E_{j}(q, \varphi)$. The electrostatic charge $q$ depends both on displacement $y$ and on superconducting phase $\varphi$.

We note at this point that we can $\operatorname{skip} \bar{E}_{c}(q)$ from the total energy and replace $E_{c}(q, \varphi)$ with $E_{j}(q, \varphi)$ even if $E_{j}(q, \varphi) \ll$ $\bar{E}_{c}(q)$. The reason is that the phase independent term $\bar{E}_{c}(q)$ results only in a small offset of $q$ from its value of $C_{g} V_{g}$. As far as the offset does not depend on phase, we can disregard it. The phase-dependent part, however, is important: it provides the coupling between the mechanical and Josephson subsystems.

To single out these contributions, we assume that (i) the voltage between the middle of the nanotube and the leads is smaller than the gate voltage, $\partial E_{c} / \partial q \ll V_{g}$, this is fulfilled if the induced charge $q \gg e$, i.e., in any practical setup; (ii) the mechanical displacement is small in comparison with the distance to the gate, $y \ll C_{g}\left(\frac{d C_{g}}{d y}\right)^{-1} \simeq L_{g}$ (in our experiments $L_{g} \simeq L \gg y$ ). With this, we linearize Eq. (6) with respect to the Josephson energy and mechanical displacement to arrive at $\left(q_{0} \equiv C_{g} V_{g}\right)$

$$
q=q_{0}+V_{g} \frac{d C_{g}}{d y} y-C_{g} \frac{\partial E_{j}}{\partial q}(q, \varphi) .
$$

The first term is the common expression for the gate-induced charge while the second and the third are the corrections of interest. At the moment, we keep $q$ in the argument of $E_{j}$, although $q \approx q_{0}$. The point is that the Josephson energy is sensitive to variations of $q$ of the order of $e$, and $\left(q-q_{0}\right)$ can, in principle, be of this order. Since $y \ll L_{g}$, we may disregard the possible $y$ dependence of $d C_{g} / d y$.

\section{B. Coupling quantities}

It is thus the charge dependence of current and force that gives rise to the coupling between the superconducting and mechanical dynamics. This dependence is, in general, complicated containing both linear and nonlinear terms. The importance of nonlinear terms is determined by comparing the resulting nonlinear feedback with intrinsic nonlinear terms characterizing the Josephson and mechanical dynamics and will be addressed in the next section. In this section, we anticipate that the charge and phase nonlinearities are unimportant and give the coupling terms linearizing the charge dependence. We need to discuss (i) the displacement dependence of current and (ii) the phase dependence of force.

(i) It is convenient to separate the superconducting current $I(q)$ into the static component $I\left(q_{0}\right)$ and the component that is linear in charge variations $(d I / d q)\left(q-q_{0}\right)$. We are interested in a $(\mathrm{dc})$ current response on the mechanical motion, the mechanical response. It arises due to the direct modulation of charge by the mechanical displacement,

$$
\tilde{I}_{\mathrm{m}}(t)=\frac{\partial I}{\partial q} \frac{d C_{g}}{d y} V_{g 0} y(t),
$$

where we used Eq. (8) to express the displacement dependence of charge.

We will mostly concentrate on the situation when the displacement oscillates at the resonant frequency, while a dc component of $\tilde{I}_{\mathrm{m}}$ is of interest. The dc signal then arises from the rectification of $y(t)$ by an oscillating part of $\partial I(\varphi) / \partial(q / e)$ that we call the detecting current.

(ii) The mechanical resonator is affected by the electrostatic force $F=-\partial E / \partial y$ :

$$
F=\frac{d C_{g}}{d y} \frac{q^{2}}{2 C_{g}^{2}},
$$

where we have used the expression of $E$ given in Eq. (7).

It is convenient to distinguish three separate contributions to the total force: the static, the gate driving, and the phasedependent Josephson forces. The static force is produced by the dc gate voltage. Its magnitude is given by $F_{\mathrm{st}}=$ $\left(d C_{g} / d y\right) V_{g 0}^{2} / 2$, corresponding to the first dominating term in Eq. (8). The effect of the static force is to tighten the resonator, thereby tuning its resonance frequency. ${ }^{15}$ Since it is stationary 
it does not excite the oscillations. The ac gate driving force arises due to the ac modulation of the gate voltage and is given by $F_{g}=\left(d C_{g} / d y\right) V_{g 0} \tilde{V}_{g} \simeq\left(q_{0} / e\right)\left(e \tilde{V}_{g} / L_{g}\right)$.

The phase-dependent Josephson force, not discussed in previous literature, comes about the product of the first and third terms in Eq. (8):

$$
F_{j}=-\frac{d C_{g}}{d y} V_{g 0} \frac{\partial E_{j}(q, \varphi)}{\partial q} .
$$

In fact, it is similar to the gate driving force, with $\tilde{V}_{g}$ replaced by the phase-dependent voltage arising in the capacitive network, $\partial E_{j}(q, \varphi) / \partial q$. In contrast to the gate driving force, the time dependence of the Josephson force is determined by the phase dynamics rather than the external modulation of the gate voltage.

The scale of the Josephson force is $\bar{F}_{j} \simeq(q / e)\left(E_{j} / L_{g}\right)$, where $E_{j}$ is the charge-dependent part of the Josephson energy, which for intermediate contact conductances $\simeq G_{Q}$ represents a fraction of $\simeq 10-50 \%$ of the total Josephson energy. The Josephson force scale can be compared to the scale of ac gate driving force, $F_{j} / F_{g} \simeq E_{j} / e \tilde{V}_{g}$. For sufficiently low ac driving amplitude $e \tilde{V}_{g} \ll E_{j}$, the Josephson force dominates $F_{g} \ll F_{j}$.

\section{Lorentz force}

The Josephson force explained above arises from the combined effect of charge sensitivity of the Josephson coupling and the capacitive coupling to the gate electrode. The alternative mechanism of generating a $\varphi$-dependent force was recently proposed by G. Sonne et al. ${ }^{21}$ This force is of Lorentz type arising from the interaction of the $\varphi$-dependent superconducting current with an external magnetic field $\vec{B}$ applied in the perpendicular direction (for the setup of Fig. 1, along the $z$ axis). This mechanism does not require the presence of a gate.

Let us compare the Lorentz force and the electrostatic Josephson force. The Lorentz force is in $y$ direction, that is, perpendicular to both $\vec{B}$ and the superconducting current, $F_{\mathrm{B}}=L|\vec{B}| I$. It is natural to express $F_{j}$ in terms of the electric field $|\vec{E}|=\left(d C_{g} / d y\right) V_{g} / C_{g}$ produced by the gate electrode. For estimates, we assume $L \simeq L_{g}$ and $\left(d C_{g} / d y\right) L / C_{g} \simeq$ $L / L_{g} \simeq 1$. This yields

$$
\frac{F_{\mathrm{B}}}{F_{j}} \simeq \frac{c|\vec{B}|}{|\vec{E}|} \alpha,
$$

where $c \simeq 3 \times 10^{8} \mathrm{~m} / \mathrm{s}$ is the speed of light and $\alpha=$ $e^{2} / 4 \pi \epsilon_{0} \hbar c \simeq 1 / 137$ is the fine structure constant.

Typical magnetic fields used in experiments are $|\vec{B}| \simeq 1 \mathrm{~T}$. They are limited from above by the critical fields of the superconducting leads. The typical electric fields are $|\vec{E}| \simeq$ $10^{7} \mathrm{~V} / \mathrm{m}$. This corresponds to a potential drop of $V_{g} \simeq 10 \mathrm{~V}$ over a distance of $L_{g} \simeq 0.5 \mu \mathrm{m}$. For these values $F_{\mathrm{B}} / F_{j} \simeq$ $10 \alpha \ll 1$ suggesting that the Josephson force dominates. Therefore, in the rest of the paper, we will disregard the Lorentz force.

If one imagines a ballistic nanotube, the Josephson coupling is not affected by the induced charge. In this case, the Lorentz force would be the only superconducting phase-dependent driving mechanism. However, the ideally ballistic nanotubes have not been realized experimentally.

\section{Analysis of nonlinearities}

Let us bring together three coupled equations governing the dynamics of the setup:

$$
\begin{gathered}
\frac{V_{b}(\omega)}{Z_{e}(\omega)}+i \frac{\hbar \omega}{2 e} \frac{\varphi(\omega)}{Z_{e}(\omega)}=[I(q, \varphi)]_{\omega}, \\
\ddot{y}+\Gamma \dot{y}+\omega_{0}^{2} y+\alpha y^{2}-\beta y^{3}=M^{-1} \frac{d C_{g}}{d y} \frac{q^{2}}{2 C_{g}^{2}}, \\
q_{0}+\frac{q_{0}}{C_{g}} \frac{d C_{g}}{d y} y-C_{g} \frac{\partial E_{j}}{\partial q}(q, \varphi)=q .
\end{gathered}
$$

The first equation describes the dynamics of superconducting phase difference $\varphi(t)$ and is obtained by applying Kirchhoff's laws to the circuit. The second equation is for the mechanical displacement $y(t)$ where we substitute the electrostatic force given in Eq. (10). The induced charge $q$ enters both equations, and at the same time is defined by the third equation, that is, its value depends both on $\varphi$ and $y$. Therefore the equations are coupled.

The equations of motion can be derived using a Lagrangian or Hamiltonian method. This we present in Appendix. We wish to simplify these equations under experimentally relevant assumptions. For this, we shall analyze the relative importance of different nonlinearities in the coupling. There are natural nonlinearity scales for all three variables: $\varphi \simeq 2 \pi, q \simeq e$, and $y \simeq y_{c} \simeq \sqrt{\omega_{0} \Gamma / \beta^{\prime}}$. This could change if the coupling is sufficiently strong. For instance, the displacement may cause the variation of phase that is subject to Josephson nonlinearity. The resulting variation of phase would produce the nonlinear variation of $q$. This will result in nonlinear feedback on $y$ and could, in principle, give rise to a nonlinear scale of $y$ that would be smaller than $y_{c}$. Therefore, first of all, we shall quantify the coupling between electrical and mechanical variables by comparing the nonlinear terms in the mechanical force resulting from the coupling with those coming from the intrinsic nonlinearities characterized by $\alpha$ and $\beta$.

The conclusion of this section is that the mechanical nonlinearity is dominant. We prove this with a rather involved reasoning given below.

For the estimations, it is convenient to introduce the following dimensionless parameters:

$$
\begin{aligned}
& A_{j}=C_{g} \frac{\partial^{2} E_{j}}{\partial q^{2}} \simeq \frac{E_{j}}{E_{C}}, \quad E_{C} \equiv \frac{e^{2}}{C_{g}}, \\
& B_{j}=\frac{2 e^{2} Z_{e}}{\hbar \omega_{0}} \frac{\partial I}{\partial q} \simeq G_{Q} Z_{e} \frac{E_{j}}{\hbar \omega_{0}} .
\end{aligned}
$$

For estimations, we assume that $A_{j}$ and $B_{j}$ are either small or of the order of 1 . This assumption is valid for $A_{j}$; it compares the Josephson energy to the charging energy under conditions of well-developed Coulomb blockade. The parameter $B_{j}$ is a coefficient of Josephson feedback at high frequency and depends on the details of the external circuit via the impedance $Z_{e}$. Unless a special effort is made to increase the circuit impedance at high frequency, $B_{j}$ will not be big.

Let us estimate the linear responses of the charge, $\delta q$, and the superconducting phase $\delta \varphi$ on a given displacement 
variation $\delta y$. We do this by expanding Eqs. (13) and (15) up to linear terms in $\delta q, \delta \varphi, \delta y$ and expressing $\delta q, \delta \varphi$ in terms of $\delta y$. This yields

$$
\begin{gathered}
\delta q\left(1+A_{j}+A_{j} B_{j}\right)=q_{0} \frac{1}{C_{g}} \frac{d C_{g}}{d y} \delta y \simeq q_{0} \frac{\delta y}{L_{g}}, \\
\delta \varphi\left(1+A_{j}+A_{j} B_{j}\right)=B_{j} \frac{q_{0}}{e} \frac{1}{C_{g}} \frac{d C_{g}}{d y} \delta y \simeq B_{j} \frac{q_{0}}{e} \frac{\delta y}{L_{g}} .
\end{gathered}
$$

Since in addition we assume that $A_{j}, B_{j} \lesssim 1$, these linear responses can be estimated as simple as

$$
\delta q \simeq q_{0} \frac{\delta y}{L_{g}}, \quad \delta \varphi \simeq B_{j} \frac{q_{0}}{e} \frac{\delta y}{L_{g}} .
$$

We use this to find a scale $y_{1}$ at which the responses of charge and superconducting phase may become comparable with the scales of their intrinsic nonlinearities $\delta q \simeq e$ and $\delta \varphi \simeq 2 \pi$. To do this, we substitute $\delta q \simeq e, \delta \varphi \simeq 2 \pi, \delta y \simeq y_{1}$. Both equations lead to the same estimation $y_{1} \simeq L_{g}\left(e / q_{0}\right)$.

We need to compare this scale with the scale $y_{c} \simeq r / \sqrt{Q}$ of the mechanical nonlinearity. This yields

$$
\frac{y_{1}}{y_{c}} \simeq \frac{e}{q_{0}} \frac{L_{g}}{r} \sqrt{Q} .
$$

The two last factors in this expression are big, while the first one can be small. We estimate the biggest $q_{0}$ from the condition that $\omega_{0}$ is changed significantly by applying the gate voltage, that is, the resonator is tightened, which leads to the estimation of the stationary displacement $y_{0} \simeq r$. This yields

$$
\frac{q_{0}}{e} \simeq r^{2} L^{1 / 2} a^{3 / 2}
$$

$a$ being atomic scale, $q_{0} / e \simeq 100$ for our devices. With this, we estimate the first two factors as $\left(e / q_{0}\right)(L / r) \simeq\left(a L / r^{2}\right)^{2}$. This is $\simeq 10$ for our geometries, and we conclude that $y_{1} / y_{c} \gg$ 1 for any $Q>1$. This implies that upon increasing the magnitude of the oscillations $\delta y$ we encounter the mechanical nonlinearity first and can disregard other nonlinearities at this magnitude scale. This proves that the dynamics of charge and phase is linear in $y$ provided our estimations of mechanical nonlinearities $\alpha$ and $\beta$ hold.

We still need to show that the coupling to Josephson junction does not change these nonlinearities significantly. To this end, we estimate the quadratic and cubic nonlinearities of the mechanical force due to coupling. First, we find the quadratic and cubic variations of the charge with respect to displacement using Eq. (15):

$$
\begin{aligned}
\delta q^{(2)} & =C_{g} \frac{\partial^{3} E_{j}}{\partial q^{3}}(\delta q)^{2} \simeq e A_{j}\left(\frac{q_{0}}{e} \frac{\delta y}{L_{g}}\right)^{2}, \\
\delta q^{(3)} & =C_{g} \frac{\partial^{4} E_{j}}{\partial q^{4}}(\delta q)^{3} \simeq e A_{j}\left(\frac{q_{0}}{e} \frac{\delta y}{L_{g}}\right)^{3} .
\end{aligned}
$$

We can now estimate the terms in the mechanical force that are quadratic and cubic in $\delta y$ :

$$
\begin{aligned}
\delta F^{(2)} & =\frac{d C_{g}}{d y} \frac{q_{0}^{2}}{2 C_{g}^{2}}\left[2\left(\frac{\delta q}{q_{0}}\right)^{2}+\frac{\delta q^{(2)}}{q_{0}}\right] \\
& \simeq F_{\mathrm{st}}\left(1+A_{j} \frac{q_{0}}{e}\right)\left(\frac{\delta y}{L_{g}}\right)^{2},
\end{aligned}
$$

$$
\begin{aligned}
\delta F^{(3)} & =\frac{d C_{g}}{d y} \frac{q_{0}^{2}}{2 C_{g}^{2}}\left(3 \frac{\delta q}{q_{0}} \frac{\delta q^{(2)}}{q_{0}}+\frac{\delta q^{(3)}}{q_{0}}\right) \\
& \simeq F_{\mathrm{st}} A_{j}\left(\frac{q_{0}}{e}\right)^{2}\left(\frac{\delta y}{L_{g}}\right)^{3} .
\end{aligned}
$$

We compare these terms with the intrinsic nonlinearities. The second-order term $\delta F^{(2)}$ needs to be compared with the mechanical quadratic nonlinearity $M \alpha \delta y^{2}$. Assuming the static displacement of the order of CNT radius, $y_{0} \simeq r$, we estimate $M \alpha \simeq M \beta / r \simeq M \omega_{0}^{2} / r$ :

$$
\frac{\delta F^{(2)}}{M \alpha \delta y^{2}} \simeq\left(1+A_{j} \frac{q_{0}}{e}\right) \frac{r^{2}}{L_{g}^{2}} \ll 1 .
$$

Here, we use the estimation $\left(q_{0} / e\right)\left(r / L_{g}\right) \ll 1$, $\left(q_{0} / e\right)\left(r / L_{g}\right) \simeq 0.1$ for typical CNT geometries [see Eq. (19)]. The third-order term $\delta F^{(3)}$ needs to be compared with the third-order nonlinearity $M \beta \delta y^{3}$. This yields

$$
\frac{\delta F^{(3)}}{M \beta \delta y^{3}} \simeq A_{j} \frac{r^{3}}{L_{g}^{3}} \ll 1 .
$$

To summarize, we proved that the nonlinear scales correspond to $\varphi \simeq 2 \pi, q \simeq e, y \simeq y_{c} \simeq \sqrt{\omega_{0} \Gamma / \beta^{\prime}}$, and that for a CNT resonator the intrinsic mechanical nonlinearities dominate the nonlinearities arising from coupling. This permits a simplification of the dynamical equations. We may linearize the terms describing the coupling of mechanical displacement and electricity, thus separating Josephson and mechanical nonlinearities.

\section{E. Workflow}

This sets the following workflow: (1) at given ac and dc bias and gate voltages, we solve for Josephson dynamics neglecting the mechanical coupling and setting $q=q_{0}(t)$. We find $I(t)$ and $\varphi(t)$. Using these, we compute the Josephson force $F_{j}$ given by Eq. (11). (2) We solve the nonlinear mechanical equation

$$
M\left(\ddot{y}+\Gamma \dot{y}+\omega_{0}^{2} y+\alpha y^{2}-\beta y^{3}\right)=F_{\text {st }}+F_{g}+F_{j}
$$

to find $y(t)$. We are mostly interested in a part that oscillates with frequency $\simeq \omega_{0}$. This may be excited by both $F_{j}$ and $F_{g}$. (3) We calculate the mechanical response current defined in Sec. III B,

$$
\tilde{I}_{\mathrm{m}}(t)=\frac{2 e}{\hbar} \frac{\partial^{2} E_{j}}{\partial \varphi \partial q} \frac{d C_{g}}{d y} V_{g 0} y(t) .
$$

(4) If we can neglect the feedback in Josephson dynamics, we are done, since the response is given directly by $\tilde{I}_{\mathrm{m}}$. In general, there is such a feedback since change of the current results in a corresponding change of phase. The condition to neglect the feedback is the condition of phase bias at frequency $\simeq \omega_{0}$, that is, the inductive impedance of the junction $\simeq \hbar \omega_{0} /\left(G_{Q} E_{j}\right)$ is much bigger than the impedance $Z_{e}\left(\omega_{0}\right)$ of the external circuit. This is the case for devices fabricated so far.

To account for the feedback, we linearize the Josephson dynamics to determine the response of the superconducting phase on the mechanical response current found, $\varphi_{\mathrm{m}}(t)$ :

$$
\varphi_{\mathrm{m}}(t)=\frac{\hbar}{2 e} \int^{t} d t^{\prime} d t^{\prime \prime} Z\left(t^{\prime}, t^{\prime \prime}\right) \tilde{I}_{\mathrm{m}}\left(t^{\prime \prime}\right)
$$


Here, the kernel $Z\left(t, t^{\prime}\right)$ represents the combined linear impedance of the external circuit and the junction. It follows from the discussion in Sec. III D that $\varphi_{\mathrm{m}}(t) \ll 2 \pi$.

(5) Taking this into account, we obtain the current response sought:

$$
I_{\mathrm{m}}=\tilde{I}_{\mathrm{m}}+\frac{2 e}{\hbar} \frac{\partial^{2} E_{j}}{\partial \varphi^{2}} \varphi_{\mathrm{m}} .
$$

The first term is the direct modulation of the current by the charge induced by the mechanical displacement while the second one is the feedback of the Josephson junction by means of $\varphi_{\mathrm{m}}$.

The mechanical response is thus typically a small correction to the maximum superconducting current. We can estimate it at maximum taking

$$
\frac{I_{\mathrm{m}}}{I_{c}} \simeq \frac{q_{0}}{e} \frac{y_{c}}{L} \simeq \frac{q_{0} r}{\sqrt{Q} e L} \simeq 10^{-3} .
$$

[We remind that $\left(q_{0} / e\right)(r / L) \simeq 0.1, Q \simeq 10^{5}$ for our devices.] Perhaps unexpectedly, the typical response becomes smaller upon increasing the quality factor. The reason for this is clear: the maximum displacement becomes smaller. However, the large $Q$ results in sharp frequency dependence of the response making it easier to identify. We thus concentrate on this dependence.

\section{F. Parameters}

Let us specify a concrete choice of the values of parameters we will use for numerical estimations. We choose these values to match those of one of the devices fabricated. Yet we shall stress that the choice made is rather arbitrary and relatively small deviations in each parameter can accumulate changing the estimations of the mechanical effect by orders of magnitude.

Junction critical current, $I_{c}: 1.0 \times 10^{-8} \mathrm{~A}$;

Total Josephson energy, $E_{j}: 2.1 \times 10^{-5} \mathrm{eV}$;

Static gate voltage, $V_{g 0}: 1.0 \mathrm{~V}$;

Static charge on the resonator, $q_{0} / e: 100$;

Resonator length and distance to gate, $L=L_{g}: 0.3 \mu \mathrm{m}$;

Resonator mass, $M$ : $4.1 \times 10^{-22} \mathrm{~kg}$;

Resonance frequency, $\omega_{0} / 2 \pi: 0.84 \mathrm{GHz}$;

Quality factor, $Q: 1.4 \times 10^{5}$;

Quadratic nonlinearity, $\alpha: 5.5 \mathrm{GHz}^{2} \mathrm{~nm}^{-1}$;

Cubic nonlinearity, $\beta: 5.5 \mathrm{GHz}^{2} \mathrm{~nm}^{-2}$;

Scale of maximum displacement, $y_{c}: 6.3 \mathrm{pm}$;

Mechanical force scale, $F_{c}: 2.3 \times 10^{-18} \mathrm{~N}$;

Josephson force, $F_{j}: 1.1 \times 10^{-15} \mathrm{~N}$.

Two important dimensionless parameters are relative value of the mechanical response current $I_{m} / I_{c}$ and the ratio of maximum Josephson force to the mechanical force scale $F_{j} / F_{c}$. As mentioned, the response is relatively small, $I_{m} / I_{c} \simeq$ $10^{-3}$. In contrast to this, $F_{j} / F_{c}$ is big, $F_{j} / F_{c} \simeq 500$.

This means that the Josephson force can easily draw the oscillator to very nonlinear regime. Let us note that the charge-dependent part of $E_{j}$ is only a fraction of the total Josephson energy, say, 10\%. This gives more realistic estimation $F_{j} / F_{c} \simeq 50$ that we will use in the plots. Besides, the oscillatory dependence of $E_{j}$ on $q$ permits tuning $F_{j}$ to zero.

\section{PHASE BIAS}

Let us start our considerations with the junction biased with a time-independent phase $\varphi$ : such bias condition can be achieved by embedding the junction into a superconducting loop. Unfortunately, our present experimental setup does not allow measurements under these bias conditions. We present the theoretical results in hope that they will be useful for future experiments.

The simplest experimental signature of Josephson force under phase bias conditions is the phase-dependent shift of the resonant frequency. The mechanism of this shift in our situation is the mechanical nonlinearity: the static Josephson force tightens or looses the nanotube resulting in the frequency change. The frequency response on the static force in our model reads

$$
\frac{d \omega_{0}}{d F}=\frac{\alpha}{M \omega_{0}^{3}} \simeq \frac{\omega_{0}}{F_{\mathrm{st}}} .
$$

The phase-dependent frequency shift reads

$$
\begin{aligned}
\Delta \omega_{0}(\varphi) & =\frac{\partial \omega_{0}}{\partial F} F_{j}(\varphi) \\
& =-\frac{\alpha}{M \omega_{0}^{3}} \frac{d C_{g}}{d y} V_{g 0} \frac{\partial E_{j}(q, \varphi)}{\partial q} \\
& \simeq \omega_{0} \frac{F_{j}(\varphi)}{F_{\mathrm{st}}} \simeq \omega_{0} \frac{E_{j}}{e V_{g 0}} \cos (\varphi) .
\end{aligned}
$$

The shift is clearly observable provided it exceeds the broadening $\Gamma$. The estimation gives

$$
\frac{\Delta \omega_{0}(\varphi)}{\Gamma} \simeq Q \frac{E_{j}}{e V_{g 0}} \cos (\varphi) .
$$

For the parameter set chosen, Eq. (29), the maximum value of the frequency shift is

$$
\left[\frac{\Delta \omega_{0}(\varphi)}{\Gamma}\right]_{\max }=2.8 .
$$

Therefore the shift is clearly observable.

Let us consider an example of a mechanically induced response under conditions of the phase bias. To excite mechanical oscillations, we apply an additional ac voltage to the gate that oscillates at the frequency $\Omega$ close to the resonant frequency $\omega_{0}$ :

$$
V_{g}=V_{g 0}+\tilde{V}_{g} \cos (\Omega t) .
$$

Assuming $\tilde{V}_{g}$ to be sufficiently small to provide a linear response of the displacement, we obtain the following expression for the resonant part of the displacement:

$$
\begin{gathered}
\tilde{y}=\frac{F_{g}}{2 M \omega_{0}} \frac{-1}{v(\varphi)+i \Gamma / 2}, \quad F_{g}=\frac{d C_{g}}{d y} V_{g 0} \tilde{V}_{g}, \\
y(t)=\frac{1}{2}\left(\tilde{y} e^{-i \Omega t}+\tilde{y}^{*} e^{i \Omega t}\right),
\end{gathered}
$$




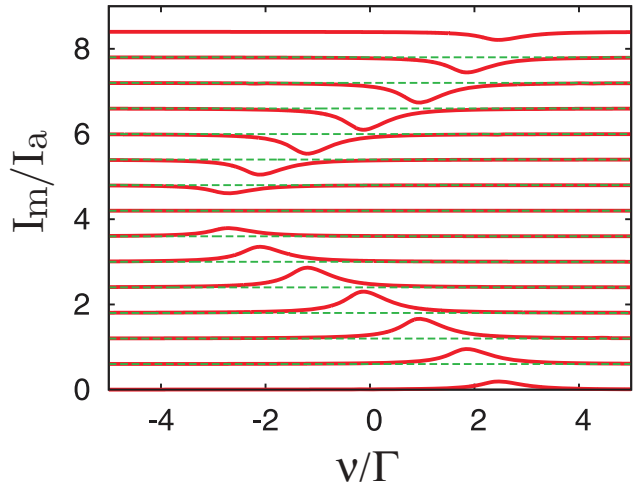

FIG. 3. (Color online) The phase-dependent frequency shift for the case of weak driving. The curves give the linear response of dc current in units $I_{a}=2 e\left(\Delta \omega_{0}\right)_{\max }|\tilde{y}|^{2}\left(M \omega_{0} / \hbar\right)$ as a function of frequency detuning for a set of phase bias values: from the lowermost to the uppermost curve the phase changes from $\varphi=\pi / 8$ to $\varphi=$ $15 \pi / 8$, with interval $\pi / 8$. The curves are offset for clarity. Dashed lines give the positions of zero.

Here, $v(\varphi)=\Omega-\omega_{0}-\Delta \omega_{0}(\varphi) \ll \omega_{0}$ is the detuning that includes the phase dependent shift of the resonance frequency discussed above. Owing to the mechanical nonlinearity, the oscillating displacement produces a stationary displacement $y=\alpha|\tilde{y}|^{2} / \omega_{0}^{2}$. This induces a stationary charge that affects the dc superconducting current at constant phase bias. Rather remarkably, this effect is related to the phase-dependent shift discussed above. Indeed, both are proportional to chargedependent part of the Josephson energy and to the nonlinearity coefficient $\alpha$. The resulting current response reads

$$
I_{m}=-2 e \frac{\partial}{\partial \varphi}\left[\Delta \omega_{0}(\varphi)\right]|\tilde{y}|^{2}\left(M \omega_{0} / \hbar\right),
$$

the contribution to the current being of the order of $e \Delta \omega_{0}$ if the displacement magnitude is of the scale of quantum fluctuations $\sqrt{\hbar / M \omega_{0}}$. The dependence on frequency of the ac modulation is a Lorentzian one, as it is frequently expected (see Fig. 3), the Lorentzian center being shifted with changing the phase.

\section{A. Fano-type response}

The above mechanism of response exploits the dominating mechanical nonlinearity. It is proportional to $\tilde{y}^{2}$. Upon increase of the ac amplitude $\tilde{V}_{g}$ the oscillating displacement saturates owing to the nonlinearities. In this case, the dominating dc current signal can arise as a result of mixing of the oscillating displacement $\tilde{y}$ and the oscillating charge $\propto \tilde{V}_{g}$. The resulting current is thus proportional to $\tilde{V}_{g} \tilde{y}$ and may exceed the contribution $\propto \tilde{y}$ provided the latter saturates.

The expression for this contribution reads

$$
I_{m}=\frac{2 e}{\hbar} \frac{\partial^{3} E_{j}(q, \varphi)}{\partial q^{2} \partial \varphi} C_{g}\left[\frac{d C_{g}}{d y} V_{g 0} \operatorname{Re}\left(\tilde{V}_{g} \tilde{y}\right)\right] .
$$

Interestingly, it exemplifies a Fano-type dependence on the detuning that is quite different from a Lorentzian. In the linear regime,

$$
I_{m}(v) \sim \frac{\Gamma}{2} \frac{v(\varphi)}{v(\varphi)^{2}+\Gamma^{2} / 4}
$$
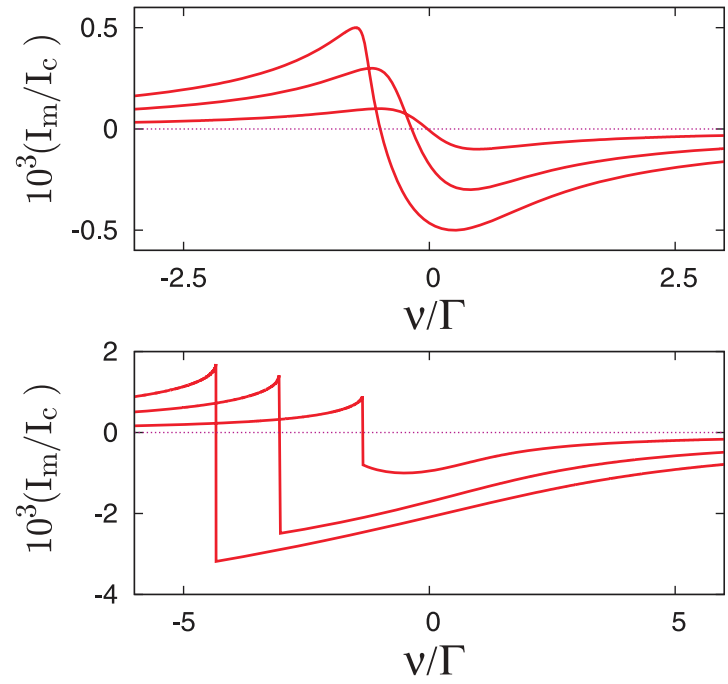

FIG. 4. (Color online) An example of Fano-type frequency dependence of the mechanical response (38). The curves correspond to the driving force values $|\tilde{F}| / F_{c}=0.2,0.6,1$ (top) and $|\tilde{F}| / F_{c}=$ $2,6,10$ (bottom). The current is in units of $\frac{d^{2} I_{1}(q)}{d q^{2}} \frac{d C_{g}}{d y} q_{0} \tilde{V}_{g} y_{c}$, which assuming $\tilde{V}_{g} / V_{g 0} \simeq 10^{-2}$ amounts to $\simeq 10^{-3} I_{c}$ for the parameter set chosen.

so that the signal changes sign at the resonance point. Figure 4 illustrates the Fano-type dependence in the nonlinear regime. Comparing expressions (37) and (39) we conclude that the Fano-shaped $I_{m}$ dominates provided $\tilde{V}_{g} / V_{g 0} \gg\left(q_{0} / e\right)^{-3 / 2} \simeq$ $10^{-3}$. The condition occurs deep in the nonlinear mechanical response regime.

\section{DIRECT CURRENT VOLTAGE BIAS}

Let us turn to dc voltage bias. In this case, the superconducting phase is, in first approximation, a linear function of time, $\varphi=\omega_{j} t, \omega_{j}=2 e V / \hbar$ being the Josephson frequency that corresponds to the voltage $V$ across the junction. In the same approximation, the current is a purely oscillatory function of time. The time-dependent current can be expanded into harmonics of the Josephson frequency,

$$
I(t)=\frac{2 e}{\hbar} \frac{\partial E_{j}}{\partial \varphi}\left(q, \varphi=\omega_{j} t\right)=\sum_{n=1} I_{n} \sin \left(n \omega_{j} t\right) .
$$

A dc current emerges from the feedback on Josephson dynamics: oscillatory currents produce oscillatory corrections to the phase. These are proportional to the impedance at frequencies $n \omega_{j}$. Taking this into account in the first approximation in $Z(\omega)$, we arrive at

$$
I_{\mathrm{dc}}=\sum_{n}\left|I_{n}\right|^{2} \frac{\operatorname{Re} Z\left(n \omega_{j}\right)}{V} .
$$

The above relation holds provided $\operatorname{Re} Z \ll V / I_{c}$. Nonperturbative treatment of Josephson dynamics is required otherwise.

Let us consider the mechanical effects. It is important to note that under the dc voltage bias the Josephson force also 
oscillates in time,

$$
\begin{aligned}
F_{j}(t) & =-\frac{d C_{g}}{d y} V_{g 0} \frac{\partial E_{j}(q, \varphi)}{\partial q} \\
& =-\frac{d C_{g}}{d y} V_{g 0} \sum_{n=1}^{\infty} \frac{\partial E_{j, n}(q)}{\partial q} \cos \left(n \omega_{j} t\right),
\end{aligned}
$$

$E_{j, n}$ being the harmonics of Josephson energy. Therefore the force can efficiently excite the mechanical resonator provided $n \omega_{j} \simeq \omega_{0}$. Let us first concentrate on the case where the resonance frequency is matched by the first harmonics, $\omega_{j} \simeq \omega_{0}$. The detuning is defined as $v=\omega_{j}-\omega_{0}$.

To start with, let us assume that the Josephson force is sufficiently weak so that the mechanical response is linear and thus, given by Eq. (35). The direct mechanical contribution to the dc Josephson current is obtained by averaging Eq. (27), and reads

$$
I_{m}=\frac{\partial I_{1}}{\partial(q / e)}\left(\frac{d C_{g}}{d y} \frac{V_{g}}{e}\right) \operatorname{Im} \tilde{y} .
$$

This can be cast to the form similar to Eq. (41),

$$
I_{m}=\left|\frac{\partial I_{1}}{\partial(q / e)}\right|^{2} \frac{\operatorname{Re} Z_{m}\left(\omega_{j}\right)}{V},
$$

where the current is replaced with detecting current $\partial I / \partial(q / e)$ and the "mechanical impedance" $Z_{m}(\omega)$ is defined as

$$
\begin{gathered}
Z_{m}(v)=\frac{\omega_{0}}{-i \nu+\Gamma / 2} Z_{m}^{(0)}, \\
Z_{m}^{(0)} \equiv \frac{\hbar}{e^{2}}\left(\frac{d C_{g}}{d y} \frac{V_{g}}{e}\right)^{2} \frac{\hbar}{2 M \omega_{0}} .
\end{gathered}
$$

(Here, $\left.v \equiv \omega_{j}-\omega_{0}.\right)$

This form of the mechanical response makes evident an analogy with Fiske steps ${ }^{25}$ that are observed at voltages corresponding to resonant frequencies of an electrical impedance. This may be either an impedance of external circuit or an effective impedance that is essentially contributed Josephson inductance.

To comprehend the scale of the response, we note first that for a sufficiently well-developed Coulomb blockade $I_{1} \simeq \partial I_{1} / \partial(q / e)$. Therefore, to compare the current given by Eq. (41) and the mechanical response, we need to compare $Z_{m}$ and a typical environmental impedance. The latter can be estimated as the impedance of free space $Z_{f} \simeq 10^{2} \mathrm{Ohm}$. The typical mechanical impedance far from the resonance $Z_{m}^{(0)}$ should be much smaller than that. Indeed, if we substitute the parameter set [see Eq. (29) in Sec. IIIF] into Eq. (46), we end up with $Z_{m}^{(0)}=0.7 \times 10^{-2} \mathrm{Ohm} \ll Z_{f}$. However, $Z_{m}$ is enhanced by a factor of $Q$ at the resonant frequency. With this, $Z_{m}>Z_{f}$ and the current peak produced by the mechanical response should exceed the background current given by Eq. (41) and be clearly observable.

The voltage dependence of dc current response in linear regime is determined by $\operatorname{Re} Z_{m}$ and thus takes a Lorentzian shape with the half-width $\delta V=V / Q$. This assumes a noiseless voltage source. It is $\mathrm{known}^{23}$ that the voltage noise suppresses the coherence of Josephson generation. For white noise spectrum of intensity $S_{V}$, the resulting linewidth reads $\delta V_{n}=(2 e / \hbar)^{2} S_{V}$. Comparing the two, we conclude that the mechanical response will be broadened by the noise and
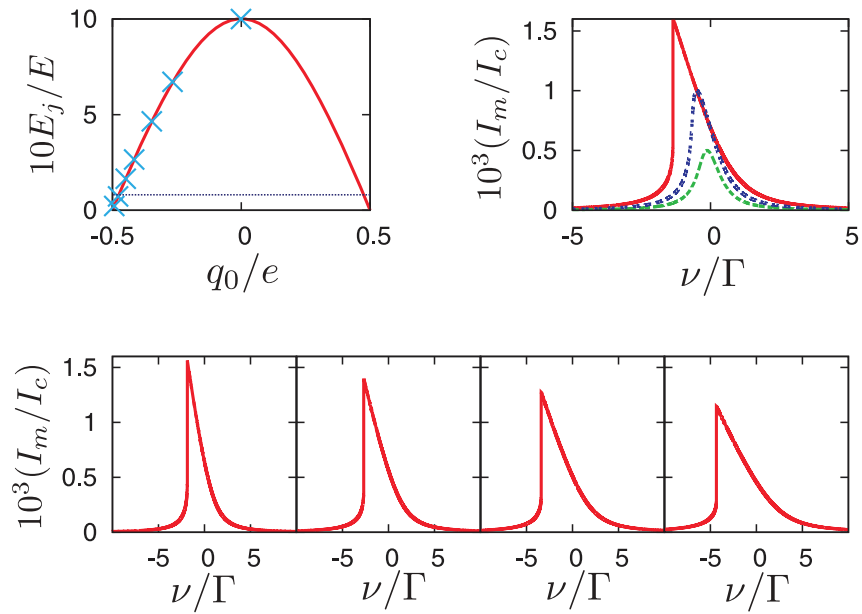

FIG. 5. (Color online) (Top left) the charge-dependent part of Josephson energy as a function of the gate-induced charge $q=C_{g} V_{g}$. The crosses indicate the values of $q$ that correspond to the values of Josephson force used in other panels. The blue dotted line indicates the value of $E_{j}$ where $F_{j}=F_{c}$. (Top right) the mechanical response as a function of detuning for relatively low values of the force $F_{j} / F_{c}<1$ at which the response increases with increasing the force. (Bottom) frequency dependence at the force values $F_{j} / F_{c}=3,5,7,10$ (from left to right ) where the response decreases with increasing the force.

essentially suppressed provided $\delta V_{n}>\delta V$, this is, $S_{V}>$ $(2 e / \hbar) \omega_{j} / Q$. Detection of the mechanical response requires $(2 e / \hbar) \omega_{j} / Q \gg S_{V}$, a condition that may be challenging to meet in practical circumstances.

As mentioned, the Josephson force can be big enough to exceed $F_{c}$, this makes it relevant to address the nonlinear response as well. We illustrate the nonlinear response in Fig. 5. In order to produce this figure, we took the charge-dependent part of the Josephson energy to be of the form $E_{j}(q, \varphi)=$ $E \cos (\varphi) \cos (\pi q / e)$. Tuning $q$ with the dc gate voltage tunes the magnitude of the Josephson force from 0 to a maximum value $\pi(q / e)\left(E / L_{g}\right)$. For illustration, we chose $E$ such that the maximum force $\pi(q / e)\left(E / L_{g}\right)=10 F_{c}(E=2.3 \mu \mathrm{eV}$ for the parameter set in use, corresponding to a $30 \%$ charge modulation of the total Josephson energy) and compute the response using Eqs. (5) and (43) at a set of the values of $q$, or, equivalently, $F_{j}$. The response is Lorentzian at small forces, increases and develops a jump characteristic for bistability. It is interesting to note that the response slowly decreases upon increasing $F_{j}$ at $F_{j}>2 F_{c}$. This is because the response is proportional to $\operatorname{Im} \tilde{y}$ that quickly decreases at big driving forces. In this limit, $I_{m} \propto F_{j}^{-1 / 3}$.

\section{A. Excitation by higher harmonics}

If we take higher harmonics of current-phase characteristic into account, we note that Josephson force emerges at a set of frequencies that are integer multipliers of $\omega_{j}$ [see Eq. (42)]. This implies that the resonant mechanical response can be also observed in the vicinities of a set of discrete voltage values satisfying $\omega_{j}=\omega_{0} / n$, this is, at lower voltages than the resonance described above. The response is computed along the same lines with replacing $E_{j, 1}$ by $E_{j, n}$. In linear 
regime, the response reads

$$
I_{m}=\left|\frac{\partial I_{n}}{\partial(q / e)}\right|^{2} \frac{n \operatorname{Re} Z_{m}\left(n \omega_{j}\right)}{V}
$$

[cf. Eq. (44), the factor $n$ in the present expression is canceled by lower voltage $\left.V=(\hbar / 2 e) \omega_{0} / n\right]$. The response scales with the relative values of the harmonics and is, in principle, of the same order of magnitude for several low harmonics. Its dependence on voltage in the vicinity of the resonance is similar to that discussed above and does not have to be illustrated separately.

\section{B. Parametric excitation}

For the sake of completeness, let us mention the possibility of the resonant mechanical response at higher voltages by means of parametric excitation. ${ }^{12}$ Generally, parametric resonance in a nonlinear oscillator is achieved by applying an ac driving force with frequency about a double of the resonant frequency, $\Omega \simeq 2 \omega_{0} .{ }^{12}$ In our case, this is achieved by applying a dc bias voltage with $\omega_{j} \simeq 2 \omega_{0}$, so that the Josephson force oscillates at $2 \omega_{0}$ and integer multiples of this frequency and thus provides the parametric driving required.

The response of at resonant frequency emerges provided the parametric driving force exceeds a certain threshold value, and, as in case of direct resonance, achieves values $\simeq y_{c}$. The point is that this threshold driving force is parametrically bigger than $F_{c}, F_{t} \simeq F_{c} \sqrt{Q}$. For our devices at $Q=10^{5}$, the parametric excitation requires Josephson energies that by a factor of 30 exceed the value from the parameter set and are not practical. This is why we do not explore the regime of parametric excitation in detail.

Besides, the manifestation of the oscillating amplitude is not as straightforward as in the case of direct resonance. The contribution of displacement at $\omega_{0}$ to the mechanical current response (27) oscillates at the same frequency and is not readily rectified to a dc current. Under our assumptions, the dc mechanical response is dominated by the displacement oscillating at $2 \omega_{0}$ and is by a factor of $\sqrt{Q}$ smaller than the typical responses studied in this paper.

\section{SHAPIRO STEPS AT RESONANT DRIVING}

From now on, we turn to the situation where the setup is ac driven at frequency $\Omega$. As discussed in Sec. II A, in our setup this gives rise to two ac signals $V_{g}(t)=\tilde{V}_{g} \cos (\Omega t+\chi)$, $V_{b}(t)=\tilde{V}_{b} \cos (\Omega t)$. The effect of $\tilde{V}_{b}$ is a formation of Shapiro steps. $^{26}$

A common approach to Shapiro steps takes into account only the first harmonics of the current-phase relation and starts with the assumption that the time-dependent superconducting phase difference can be presented as a sum of three terms:

$$
\varphi(t)=\varphi_{1} \sin (\Omega t)+\omega_{j} t+\varphi_{0}
$$

Here, the first term describes the ac driving $\varphi_{1}>0, \varphi_{1}=$ $\left|\tilde{V}_{b}\right| /(2 e / \hbar) \Omega$, the second term corresponds to a dc voltage $V=\omega_{j} /(2 e / \hbar)$, and the third term is a lock-in phase important for further consideration. With this, $\sin (\varphi)$ can be presented as a sum over harmonics:

$$
\sin (\varphi)=\sum_{m=-\infty}^{\infty} J_{m}\left(\varphi_{1}\right) \sin \left(\Omega_{m} t+\varphi_{0}\right)
$$

with $\Omega_{m}=m \Omega+\omega_{j}$. Here, $J_{m}$ denotes the $m$ th Bessel function of the first kind.

Shapiro steps are formed at discrete values of dc voltage $\left|\omega_{j}\right|=m \Omega$. In this case, the time-dependent current $I(t)=$ $I_{c} \sin (\varphi(t))$ has a dc component

$$
I_{\mathrm{dc}}=-I_{c} \operatorname{sgn}(V) J_{m}\left(\varphi_{1}\right) \sin \varphi_{0} .
$$

The simplest assumption is an ideal current bias at zero frequency and an ideal voltage bias at frequencies $\simeq \Omega$. In this case, the $I-V$ curve of ac driven junction consists of a series of separate pieces. At each piece (Shapiro step), the voltage is locked to one of the discrete values. The current within each piece may vary from minimum values $I_{-}$to the maximum value $I_{+}$provided the bias current fits this interval. In this case, the actual value of the lock-in phase $\varphi_{0}$ is set by the bias current. The extremal values $I_{ \pm}= \pm I_{c}\left|J_{m}\left(\varphi_{1}\right)\right|$ are achieved at the lock-in phases given by

$$
\varphi_{0}^{ \pm}=\mp \pi / 2 \operatorname{sgn}\left[V J_{m}\left(\varphi_{1}\right)\right] .
$$

We mostly follow this approach, while admitting extreme simplifications it brings. The higher harmonics of currentphase relation and/or nonideal voltage bias not only modify the relation between the current and lock-in phase: they also provide phase locking at fractional ratios of $\omega_{j} / \Omega^{28}$ and formally at all rational values of this ratio. These fractional Shapiro steps are, however, more sensitive to noise than the integer ones and more likely to vanish. The $I-V$ curves of our devices do show well-developed steps at integer values of $\omega_{j}$ and only traces of phase-locking at intermediate values. For this reason, we do not consider fractional Shapiro steps in this paper and concentrate on integer ones where $\left|\omega_{j}\right|=m \Omega$.

It is advantageous to look at the mechanical response at Shapiro steps rather than at dc bias conditions. The external ac driving synchronizes Josephson oscillations. The inductive response present at Shapiro steps also reduces significantly the voltage noise at low frequencies so that it does not broaden the resonant lines. In this section, we will consider the mechanical response in the simplest situation of resonant driving where the driving frequency matches the resonant frequency, $\Omega \simeq \omega_{0}$.

\section{A. First step}

Let us first concentrate on the first Shapiro step, the one at voltage $2 \mathrm{eV} / \hbar= \pm \Omega$, that is the biggest in the limit of small driving voltages $\varphi_{1} \ll 1$, and determine the dc part of the response at the oscillating displacement $\tilde{y}$. To represent the results, we normalize $\tilde{y}$ to the nonlinearity scale $y_{c}$ and introduce a convenient current scale:

$$
\bar{I}=\frac{\partial I_{1}}{\partial q} \frac{d C_{g}}{d y} V_{g 0} y_{c} .
$$

For the values of our parameter set,

$$
\bar{I} \simeq I_{c}\left(q_{0} / e\right)\left(y_{c} / L_{g}\right)=2.1 \times 10^{-3} I_{c} .
$$


Making use of Eqs. (27) and (49), we express the mechanical response in terms of the amplitudes $\tilde{y}$ and $\bar{I} j$ at the resonant frequency,

$$
\begin{gathered}
I_{m}=\frac{\bar{I}}{y_{c}} \operatorname{Re}\left(j^{*} \tilde{y}\right), \\
j=-i \begin{cases}\left(J_{2}\left(\varphi_{1}\right) e^{i \varphi_{0}}-J_{0}\left(\varphi_{1}\right) e^{-i \varphi_{0}}\right) & \text { if } V>0, \\
\left(J_{0}\left(\varphi_{1}\right) e^{i \varphi_{0}}-J_{2}\left(\varphi_{1}\right) e^{-i \varphi_{0}}\right) & \text { if } V<0 .\end{cases}
\end{gathered}
$$

This displacement is a response on the force at resonant frequency, which is a sum of Josephson force and gate force. The time-dependent Josephson force is expanded in harmonics in the form

$$
\begin{aligned}
F_{j}(\varphi) & =\bar{F}_{j} \cos [\varphi(t)], \\
& =\bar{F}_{j} \sum_{m=-\infty}^{\infty} J_{m}\left(\varphi_{1}\right) \cos \left(\Omega_{m} t+\varphi_{0}\right), \\
\bar{F}_{j} & =-\frac{d C_{g}}{d y} V_{g 0} \frac{\partial E_{1, j}(q)}{\partial q} \simeq \frac{q_{0}}{e} \frac{E_{j}}{L_{g}} .
\end{aligned}
$$

Its amplitude at resonant frequency is contributed by the terms $m=0,2$ and reads

$$
\begin{gathered}
\tilde{F}_{j}=\bar{F} f, \\
f= \begin{cases}\left(J_{2}\left(\varphi_{1}\right) e^{i \varphi_{0}}+J_{0}\left(\varphi_{1}\right) e^{-i \varphi_{0}}\right) & \text { if } V>0, \\
\left(J_{0}\left(\varphi_{1}\right) e^{i \varphi_{0}}+J_{2}\left(\varphi_{1}\right) e^{-i \varphi_{0}}\right) & \text { if } V<0 .\end{cases}
\end{gathered}
$$

Let us discuss first the relative scale of the gate force in comparison with the Josephson force. It may seem there is none, and varying the ac gate voltage $\tilde{V}_{g}$ one can achieve any ratio $\simeq \tilde{V}_{g} / E_{J}$ between the forces. However, we should take into account the fact that in our setups ac driving also induces an appreciable bias voltage $\tilde{V}_{b}$. If the oscillating phase $\varphi_{1}$ produced by this voltage becomes large Shapiro steps can hardly be observed. It is, in general, reasonable to expect $\tilde{V}_{g} \simeq$ $\tilde{V}_{b}$. In this case, $\varphi_{1} \simeq 1$ corresponds to $F_{g} / F_{j} \simeq \hbar \omega_{0} / E_{j}$. The latter ratio is typically $10^{-2}$ in our setups (for our parameter set, it is $\hbar \omega_{0} / E_{j}=2.7 \times 10^{-2}$ ). This implies that typically we can disregard ac gate force in comparison to the Josephson force. We will analyze this case first and consider the effect of the gate force in the end of the section.

With this, the mechanical response is given by

$$
\begin{aligned}
I_{m}= & \frac{\bar{I} \bar{F}}{F_{c}} \operatorname{Re}\left(j^{*} f R\right) \\
= & \frac{\bar{I} \bar{F}}{F_{c}}\left\{\operatorname{sgn} V\left[J_{0}^{2}\left(\varphi_{1}\right)-J_{2}^{2}\left(\varphi_{1}\right)\right] \operatorname{Im}(R)\right. \\
& \left.-J_{0}\left(\varphi_{1}\right) J_{2}\left(\varphi_{1}\right) \sin \left(2 \varphi_{0}\right) \operatorname{Re}(R)\right\} .
\end{aligned}
$$

Here, $R \equiv R\left(v /(\Gamma / 2),\left(F_{j} / F_{c}\right)|f|\right)$ defined by Eq. (5) gives the nonlinear mechanical response. The expression is naturally separated onto two terms. The first term is proportional to $\operatorname{Im}(R)$ and therefore exhibits a Lorentz-like dependence on frequency. It does not depend on the lock-in phase and can be regarded as a shift in the current. Owing to the shift, the maximum and minimum currents $I^{ \pm}$at the step are no more opposite: the mechanical effect breaks the symmetry of the Shapiro step. The shift is, however, opposite for opposite voltages. The origin of the shift may be traced to the Fiske response [see Eq. (44)] formed at $\omega_{j} \simeq \omega_{0}$ in the absence of the ac driving. Indeed, in the limit of vanishing $\varphi_{1}$, the first term in the mechanical response does not vanish: rather, it approaches the expression (44). So it looks like the Fiske response persists also for well-developed Shapiro steps and contributes to the current at the step. This suggest perhaps the easiest way to observe and identify the mechanical response: measure maximal and minimal currents at a step as function of the ac frequency. In the rest of the paper, we thus mainly concentrate on the modification of extremum currents.

The second term in Eq. (58) cannot, however, be observed in this way. In ideal current bias conditions at low frequency, the second term in the current response fact amounts to a shift of the lock-in phase. Indeed, since the current at the step as a function of $\varphi_{0}$ reads as $I(\varphi)=-\operatorname{sgn}(V) J_{1}\left(\varphi_{1}\right)$, the second term can be seen as a modification of the lock-in phase at constant bias current that does not depend on this current,

$$
\left(\Delta \varphi_{0}\right)_{\mathrm{m}}=-\operatorname{sgn}(V) \frac{\bar{I} \bar{F}}{I_{c} F_{c}} \frac{J_{0}\left(\varphi_{1}\right) J_{2}\left(\varphi_{1}\right)}{J_{1}\left(\varphi_{1}\right)} \operatorname{Re}(R) .
$$

This response is of Fano type. Since such shift of the phase does not modify the values of the current extrema, the effect cannot be observed in the course of two-terminal electrical measurement in our setup. The shift of the lock-in phase can be however revealed if the Josephson junction under consideration is a part of a superconducting quantum interference device (SQUID), or with the aid of lock-in measurement at nonresonant ac frequency.

With respect to this, we ought to mention yet another effect of Josephson force manifesting itself in the mechanical response considered. In fact, the situation at a Shapiro step is similar to the phase bias conditions considered in Sec. IV, with lock-in phase playing the role of $\varphi$. We thus expect $\varphi_{0}$-dependent shift of the resonance frequency. The static Josephson force at a Shapiro step is given by

$$
F_{j}=\bar{F} \operatorname{sgn}(V) J_{1}(\varphi) \cos \left(\varphi_{0}\right) .
$$

The frequency shift caused by this force thus reads

$$
\Delta \omega_{0}\left(\varphi_{0}\right)=\operatorname{sgn}(V)\left(\Delta \omega_{0}\right)_{\max } J_{1}(\varphi) \cos \left(\varphi_{0}\right) .
$$

Here, $\left(\Delta \omega_{0}\right)_{\max }$ is a maximum frequency shift in the absence of the ac driving, given by Eq. (31). The frequency shift vanishes at extremum points $\varphi_{0}^{ \pm}$and therefore cannot be observed by measuring the extrema of the current.

We illustrate the mechanical response in Fig. 6. In this figure as well in all subsequent figures except Fig. 7, we concentrate on the modification of the maximum current on the step. Instead of presenting the (rather trivial Lorentz like) frequency dependence of this modification, we give the extremum of this modification over the frequency range and plot it versus $\varphi_{1}$. The extremum is proportional to the maximum of $\operatorname{Im}(R)$ over the frequency. We shall note that the dependence of this maximum on the force is rather specific one: it is a constant until the bistability threshold $F=1.24 F_{c}$, has a cusp at this value of force, and decreases monotonously at higher forces. This accounts for rather strange appearance of the response curves. If the Josephson force is smaller than the bistability threshold, they coincide with the linear response given by the dotted curves. Otherwise, the response is smaller than linear one and exhibits kinks. 


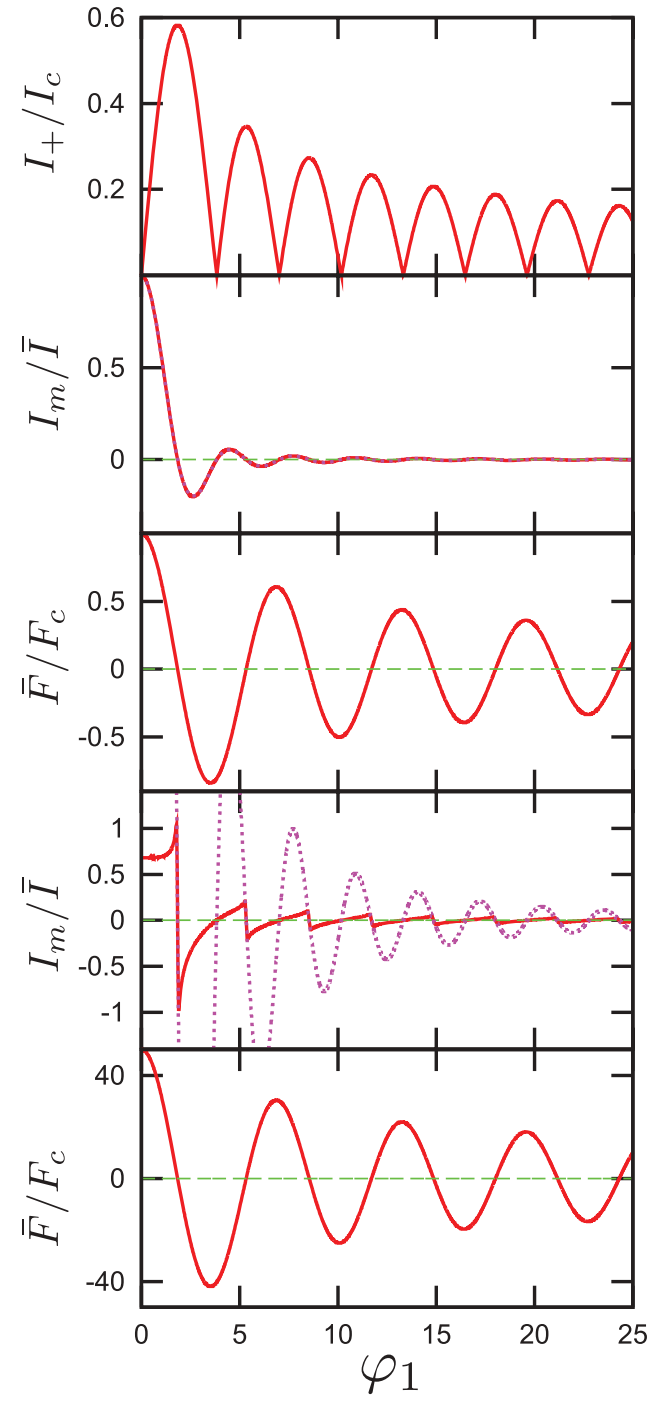

FIG. 6. (Color online) The mechanical response at resonant driving $\Omega \simeq \omega_{0}$ and at the first Shapiro step $V_{0}=2 e \Omega / \hbar$ vs the oscillating phase $\varphi_{1}$. The first (upper) plot gives the maximum current at the step. The second and fourth plots give the mechanical response defined as the extremum of the modification of this maximum current over the frequencies near the resonance, at maximum Josephson forces $\bar{F}=F_{c}$ and $\bar{F}=50 F_{c}$, respectively. The actual amplitudes of the resonant Josephson forces are given at the lower plots, respectively third and fifth.

The so-defined maximum response is plotted in Fig. 6 for two values of $\bar{F}$, those correspond to slightly and strongly nonlinear regime, respectively. In both cases, the response vanishes when the width of Shapiro step reaches maximum, or becomes zero (except $\varphi_{1}=0$ ). In slightly nonlinear regime, the response reaches maximum value at $\varphi_{0}$. Upon increasing $\varphi_{1}$, it exhibits Bessel-like oscillations. The envelop of these oscillations shrinks with increasing $\varphi_{1}$. This shrinking is much faster than that for either step width or Josephson force. In strongly nonlinear regime, the amplitude of the response is determined by competition of two factors: it is increased by the bigger value of the detecting current, and decreased owing to smaller imaginary part of oscillating displacement at higher Josephson forces.
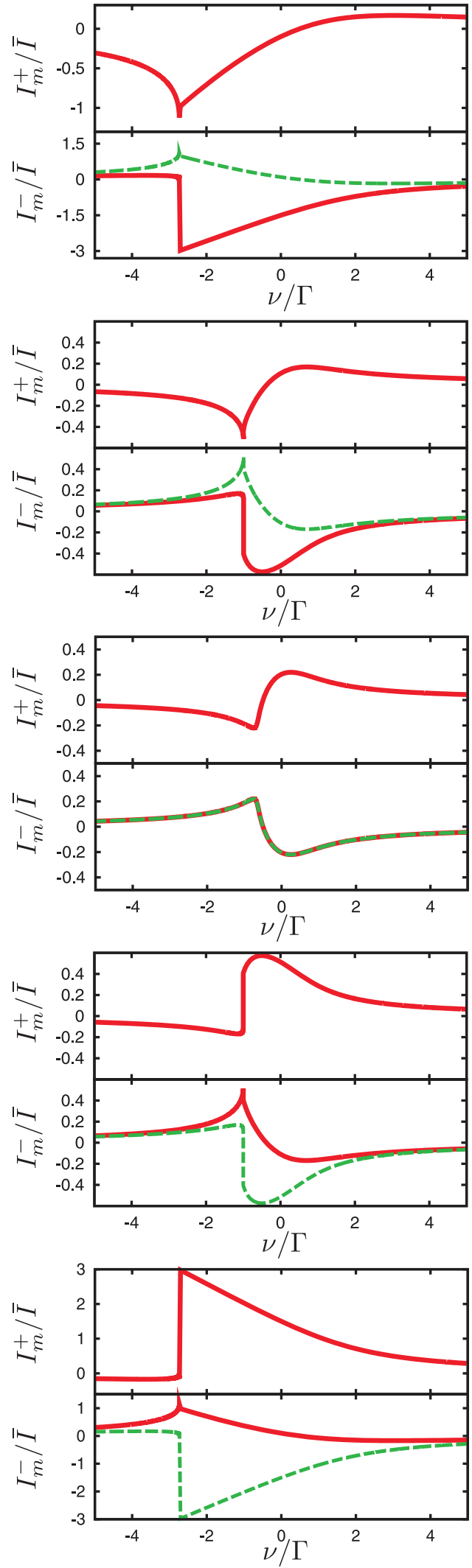

FIG. 7. (Color online) The effect of gate force. For all plots, the gate force is fixed to $F_{g}=F_{c}$. In the plots from top to bottom, the maximum Josephson force $\bar{F}$ assumes the values $\bar{F} / F_{c}=-5$, $-1,0,1,5$. We choose $\chi=0$ and $\varphi_{1}=1$. Dashed lines in the plots for $I^{-}$give values opposite to the corresponding $I^{+}$, to stress the symmetry or asymmetry of the response. 
Let us turn to the effect of the gate force. The Josephson force of the kind considered can change sign and therefore be tuned to zero by tuning $q$. In the vicinity of this particular $q$, the gate force should compete with the Josephson one and eventually dominate. The full amplitude of the force at the resonant frequency then reads

$$
\tilde{F}=\bar{F} f+F_{g} \exp (-i \chi),
$$

the frequency shift $\chi$ between the bias and the gate voltage being a relevant parameter.

The mechanical response is given by Eq. (58) where $R$ depends on the full force plus an addition proportional to $F_{g}$,

$$
\begin{aligned}
I_{m}^{(g)}= & \frac{\bar{I} F_{g}}{F_{c}} \operatorname{Re}\left[j^{*} \exp (-i \chi) R\right] \\
= & \frac{\bar{I} \bar{F}}{F_{c}}\left\{-\left[J_{0}\left(\varphi_{1}\right) \sin \left(\varphi_{0}+\chi\right)+J_{2}\left(\varphi_{1}\right) \sin \left(\varphi_{0}-\chi\right)\right]\right. \\
& \times \operatorname{Re}(R)\left[J_{0}\left(\varphi_{1}\right) \cos \left(\varphi_{0}+\chi\right)-J_{2}\left(\varphi_{1}\right) \cos \left(\varphi_{0}-\chi\right)\right] \\
& \times \operatorname{Im}(R)\} .
\end{aligned}
$$

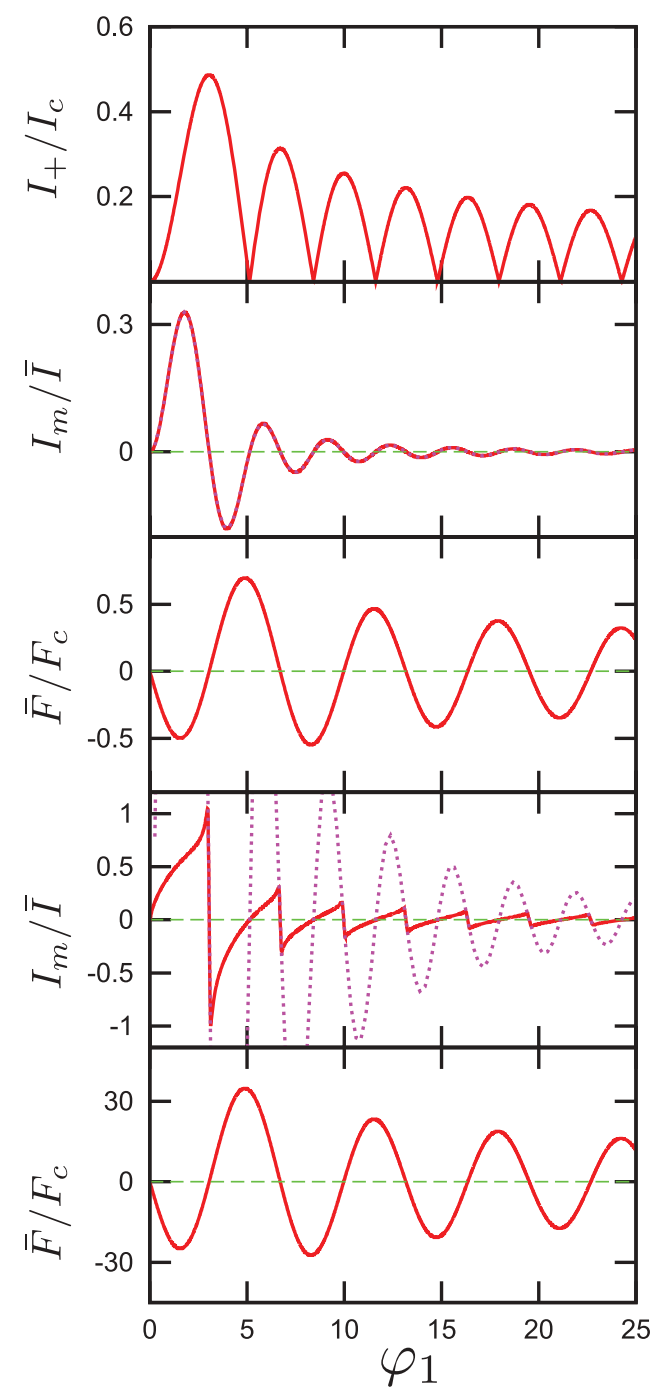

FIG. 8. (Color online) The maximum current at the Shapiro step, the mechanical response and the force versus $\varphi_{1}$ for $\bar{F}=F_{c}$ and $\bar{F}=50 F_{c}$ at the second Shapiro step $V_{0}=4 e \Omega / \hbar$.
The last equation holds for $V>0$. The expression for $V<0$ is obtained by interchanging $J_{0}$ and $J_{2}$. Evaluating this at the extremum points of lock-in phase, $\varphi_{0}^{ \pm}$, we obtain

$$
\begin{aligned}
I_{m}^{(g) \pm}= & \mp \operatorname{sgn}\left[V J_{1}\left(\varphi_{1}\right)\right] \frac{\bar{I} F_{g}}{F_{c}} \\
& \times\left[J_{0}\left(\varphi_{1}\right)+J_{2}\left(\varphi_{1}\right)\right] \operatorname{Re}[R \exp (-i \chi)] .
\end{aligned}
$$

Therefore the contribution of the gate force to extremum currents is not like a shifts; rather, it modifies the width of the step. These terms are even in voltage and display a mixture of Fano-type and Lorentzian-type response as a function of frequency, this being tuned by the phase $\chi$.

To illustrate a rather complex interplay of Josephson and Shapiro dynamics, we plot in Fig. 7 the frequency dependence of the mechanical response for a constant $V_{g}$ and a set of values of $F_{j}$ that pass zero. The plots show the modifications of extremum currents $I^{ \pm}$. These modifications are the same for the Josephson force contribution and opposite for the gate force contribution. Besides, the frequency dependence is Fano like for the gate force contribution and Lorenz like

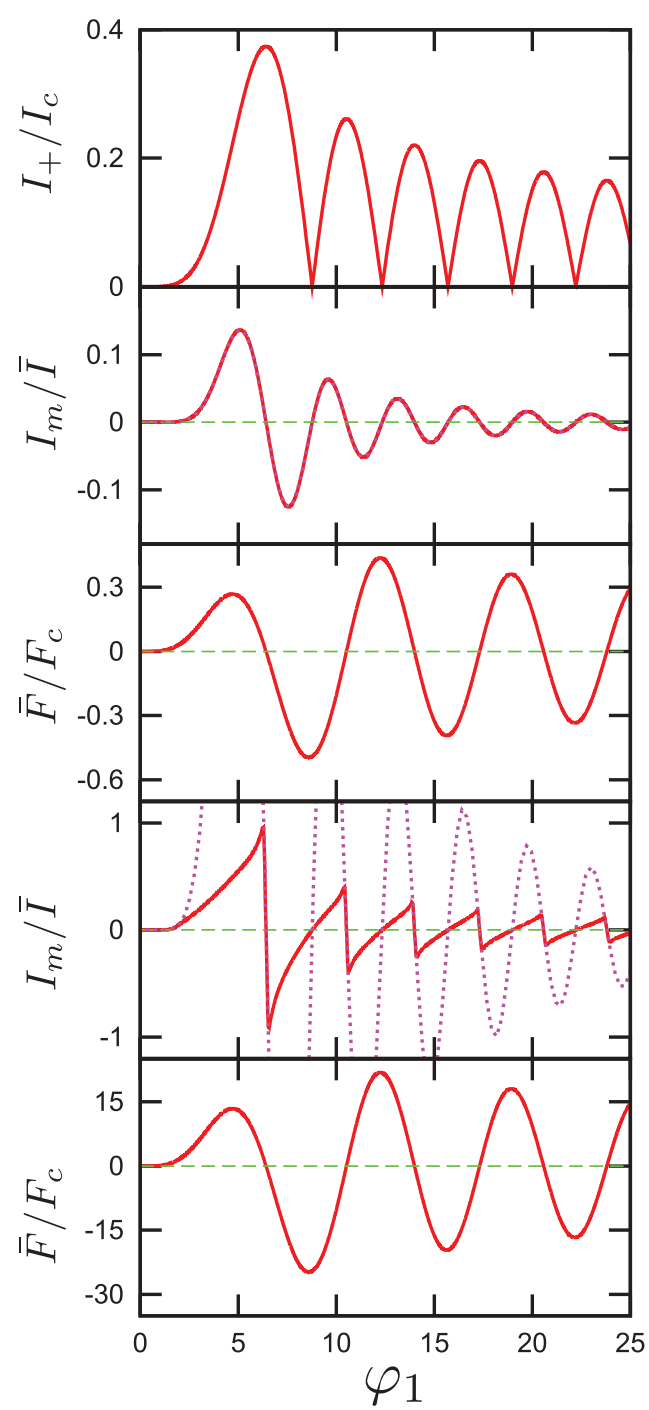

FIG. 9. (Color online) The same as in Fig. 8 at the fifth Shapiro step $V_{0}=10 e \Omega / \hbar$. 
for the Josephson force contribution. In the central plot, the Josephson force contribution is absent, the modifications of $I^{ \pm}$are opposite, and the frequency dependence is Fano like. Upon increasing the Josephson force, these features are transformed into the opposite ones. The plots are symmetric upon simultaneous change of signs of the current and the Josephson force.

\section{B. Higher steps}

At the same conditions of the resonant driving, we analyze the mechanical response at other Shapiro steps $m>1$, those correspond to higher voltages $|V|=m \hbar \omega_{0} / 2 e$. Both the amplitudes of the detecting current and the Josephson force display a complex dependence on the step number $m$ and the oscillating phase $\varphi_{1}$. They are given by

$$
\begin{aligned}
& j=-i\left(J_{-1+\bar{m}} e^{i \varphi_{0}}-J_{1+\bar{m}} e^{-i \varphi_{0}}\right), \\
& f=J_{-1+\bar{m}} e^{i \varphi_{0}}+J_{1+\bar{m}} e^{-i \varphi_{0}},
\end{aligned}
$$

where the dependence on the sign of the voltage is incorporated into $\bar{m} \equiv-\operatorname{sgn}(V) m$. We consider only the situation when Josephson force dominates. With this, we obtain a relation similar to Eq. (58):

$$
\begin{aligned}
I_{m}= & \frac{\bar{I} \bar{F}}{F_{c}} \operatorname{Re}\left(j^{*} f R\right) \\
= & \frac{\bar{I} \bar{F}}{F_{c}}\left\{\operatorname{sgn} V\left[J_{m-1}^{2}\left(\varphi_{1}\right)-J_{m+1}^{2}\left(\varphi_{1}\right)\right] \operatorname{Im}(R)\right. \\
& \left.-J_{m-1}\left(\varphi_{1}\right) J_{m+1}\left(\varphi_{1}\right) \sin \left(2 \varphi_{0}\right) \operatorname{Re}(R)\right\} .
\end{aligned}
$$

As in the first step, the response consists of two terms. The first one gives a shift in the current, and gives a modification of the maximum and minimum currents at the step, this is to be measured. As in the previous case, the shift is odd in voltage. However, its $\varphi_{1}$ dependence is quite rather distinct.
The measuring of the mechanical response at higher steps is important to check the consistency of results and thereby unambiguously identify the mechanism of the response. The characteristic dependencies on $\varphi_{1}$ make the identification easy.

We illustrate the response for higher steps in Figs. 8 (second step) and 9 (fifth step). In both cases, the response correlates with the Shapiro step width given in the upper plots: it vanishes when the width achieves a maximum or becomes zero. In distinction from the first step, the responses vanish at vanishing $\varphi_{1}$. Their typical values are of the same order. However, the envelopes of the responses decrease rather slow with increasing $\varphi_{1}$.

\section{SHAPIRO STEPS AT NONRESONANT DRIVING}

In the previous section, we concentrate on the case when the driving frequency $\Omega$ matches the resonant frequency of the mechanical oscillator. It is not a necessary condition for an efficient excitation of the resonant mode. The Josephson dynamics at Shapiro steps is essentially nonlinear. As a consequence, the spectrum of current oscillations contains all higher harmonics $n \Omega$ of the driving frequency $\Omega$. The same pertains to the Josephson force. Therefore the resonator can be efficiently excited for $\Omega=\omega_{0} / N, N>1$ being an integer number. At any given $N$, the resonant conditions are achieved for any Shapiro step number $m$, and thus for voltages $2 e V / \hbar=\omega_{j}=(m / N) \omega_{0}$.

These nonresonant driving conditions are advantageous for observation of the Josephson force since the ac gate voltage force is not in the resonance, does not cause any appreciable displacement and therefore does not mask the effect of the Josephson force. In this short section, we will thus concentrate on the case of the nonresonant driving $\Omega=\omega_{0} / N$.

The amplitudes of the detecting current and the Josephson force depended not only on the step number $m$ and the
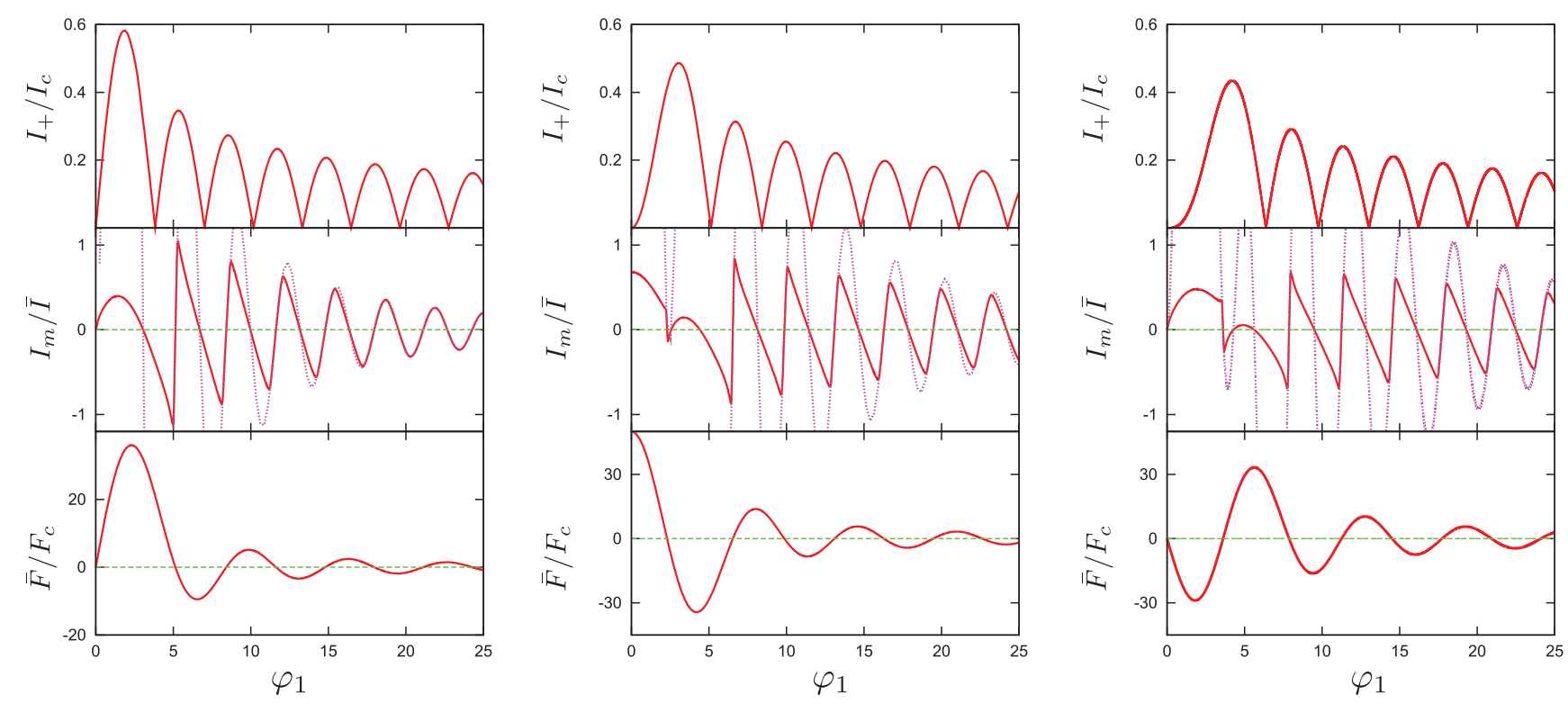

FIG. 10. (Color online) The mechanical response at the nonresonant driving. Here, the ac driving frequency is $\Omega \simeq \omega_{0} / 2$, corresponding to $N=2$. From left to right, the three columns correspond to Shapiro steps $m=1,2,3$. Plotted are the maximum current at the Shapiro step, the mechanical response and the amplitude of the force. The maximum of the Josephson force was set to $\bar{F}=50 F_{c}$ for all plots. 

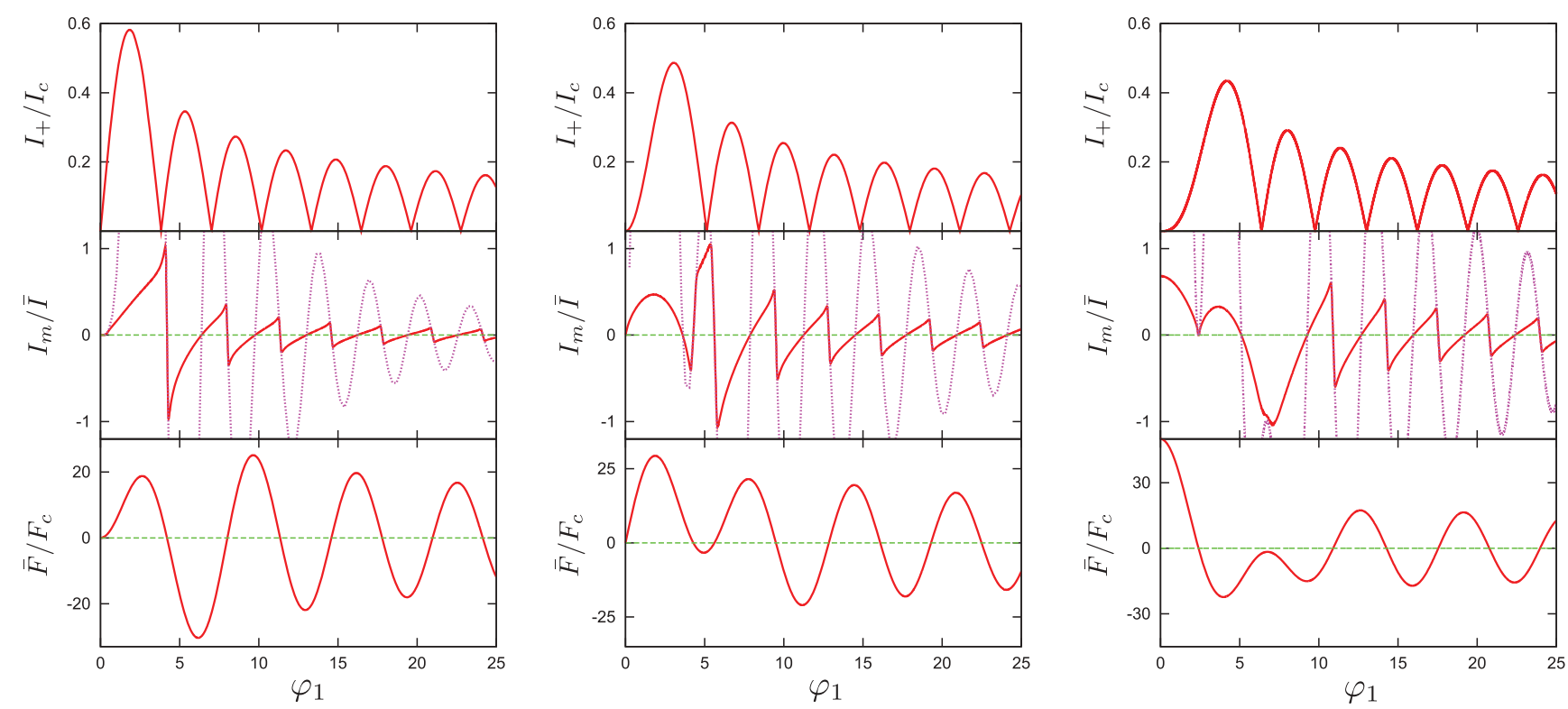

FIG. 11. (Color online) The mechanical response at the nonresonant driving for $N=3$. Except this, all other parameters are the same as in Fig. 10.

oscillating phase $\varphi_{1}$, but also on $N$. They are given by

$$
\begin{aligned}
& j=-i\left(J_{-N+\bar{m}} e^{i \varphi_{0}}-J_{N+\bar{m}} e^{-i \varphi_{0}}\right), \\
& f=J_{-N+\bar{m}} e^{i \varphi_{0}}+J_{N+\bar{m}} e^{-i \varphi_{0}},
\end{aligned}
$$

where the dependence on the sign of the voltage is again incorporated into $\bar{m} \equiv-\operatorname{sgn}(V) m$.

Since the gate force is absent, the response is given by a relation similar to Eq. (58) that contains the Josephson force only:

$$
\begin{aligned}
I_{m}= & \frac{\bar{I} \bar{F}}{F_{c}} \operatorname{Re}\left(j^{*} f R\right) \\
= & \frac{\bar{I} \bar{F}}{F_{c}}\left\{\operatorname{sgn} V\left[J_{m-N}^{2}\left(\varphi_{1}\right)-J_{m+N}^{2}\left(\varphi_{1}\right)\right] \operatorname{Im}(R)\right. \\
& \left.-J_{m-N}\left(\varphi_{1}\right) J_{m+N}\left(\varphi_{1}\right) \sin \left(2 \varphi_{0}\right) \operatorname{Re}(R)\right\} .
\end{aligned}
$$

It is again separated onto two terms discussed above, only the first term being responsible for the modification of the extremum currents of the Shapiro steps.

We illustrate the dependencies on $\varphi_{1}$ in Figs. 10 (for $N=2$ ) and 11 (for $N=3$ ) for the first three steps with $m=1,2,3$. The vertical arrangement of the plots is the same as in the previous figures except we chose a single value of the maximum Josephson force $\bar{F}=50 F_{c}$ that brings us deep into the nonlinear regime. In this regime, the response is of the same order of magnitude for all steps and ratios $N$, while retaining unique $m, N$ specific dependence on $\varphi$ that can be used for identification of the effect and the characterization of the Josephson force.

\section{CONCLUSIONS}

In conclusion, we have studied Josephson junction dynamics affected by excitation of a mechanical resonator. We have demonstrated that the mechanical oscillations can be rectified giving rise to an additional dc current that can be used for detection. The mechanical response is proportional to the oscillation amplitude, and is estimated as $I_{m} \simeq I_{c}\left(q_{0} / e\right)(y / L) \simeq 10^{-3} I_{c}\left(y / y_{c}\right)$. The resonator can be driven by the ac voltage applied to the gate electrode as well as an additional mechanical force, termed the Josephson force, that depends on the superconducting phase difference at the junction. We estimate the Josephson force as $\bar{F}_{j} \simeq$ $\left(q_{0} / e\right)\left(E_{j} / L_{g}\right)$ and show that it is sufficiently strong to drive the mechanical resonator into the nonlinear regime. We also show that it is typically larger than magneto-induced force proposed in Ref. 21.

We have presented a general and detailed analysis of the coupling between electrical and mechanical degrees of freedom, discussing the competing nonlinearities. This analysis is applied to a Josephson device with a suspended CNT resonator, where we show that the intrinsic nonlinearity scales dominate those arising from the coupling.

We have provided analytical formulas for the response of the device to mechanical excitations in a wide interval of the excitation strengths and for various biasing schemes. We discuss distinct frequency dependencies, Lorentz and Fano-like, of the mechanical response both for linear and nonlinear regimes and show how these arise based on the nature of the resonant mechanical force. In the case of a phase biased junction, we show that the resonant frequency of the mechanical mode acquires a measurable phase-dependent shift (see Fig. 3).

We have discussed conditions of detecting the enhanced mechanical response arising when the Josephson frequency matches the resonance frequency of the mechanical mode. We reasoned that the regime of Shapiro steps is advantageous, since the fluctuations of the voltage drop over the junction are suppressed. We provided expressions for the mechanical response in the regime of Shapiro steps and demonstrated that it manifests as modifications of the extrema of the steps. We show that the mechanical mode can be efficiently excited 
not only by resonant ac signals, but also by ac signals with frequencies close to an integer fraction of the mechanical resonance frequency. Our preliminary experimental results confirm this behavior; these will be reported elsewhere.

\section{ACKNOWLEDGMENTS}

The authors would like to thank G. A. Steele, S. M. Frolov, L. P. Kouwenhoven, and H. S. J. van der Zant for many useful discussions. This research was supported by the Dutch Science Foundation NWO/FOM.

\section{APPENDIX: LAGRANGIAN FORMALISM}

Here, we present a derivation of the equations of motion using the Langrangian of our junction model, describing the dynamics of the three variables $y, q$, and $\varphi$, treated here as generalized degrees of freedom. To account for effects of dissipation, we introduce generalized friction forces for each degree of freedom and treat these as external forces.

The kinetic energy of the system is that of the mechanical resonator:

$$
T=\frac{1}{2} M \dot{y}^{2} .
$$

The inertial effects associated with variations of $\varphi$, in the main text, we have conveniently treated those using the external impedance $Z_{e}$, which describes both inertial effects as well as dissipation. We do the same here treating both effects as a generalized external force proportional to $Z_{e}$.

The potential energy includes contributions from the mechanical, electrostatic, and Josephson energies $U=U_{m}+$ $U_{e}+E_{j}$ :

$$
\begin{gathered}
U_{m}=\frac{1}{2} M \omega_{0}^{2} y^{2}-M \frac{\alpha}{3} y^{3}-M \frac{\beta}{4} y^{4}, \\
U_{e}=\bar{E}_{c}(q)+\frac{q^{2}}{2 C_{g}(y)}-q V_{g} .
\end{gathered}
$$

The system Lagrangian is given by $\mathcal{L}=T-U$. Equations of motion are obtained using

$$
\frac{d}{d t}\left(\frac{\partial T}{\partial \dot{x}}\right)-\frac{\partial T}{\partial x}=-\frac{\partial U}{\partial x}+F_{x},
$$

where $x=y, g, \varphi$ denotes a generalized degree of freedom and $F_{x}$ is the corresponding generalized friction force. The mechanical friction force is modeled typically as linear in variation of displacement $F_{y}=-M \Gamma \dot{y}$.

Dissipative effects associated to charging of the gate capacitance are important on timescale $R_{g} C_{g}$, where $R_{g}$ is the negligible resistance of wires grounding the gate and source-drain electrodes. We assume that $R_{g} C_{g} \ll \omega_{0}^{-1}$, such that the effect of gate charging on the dynamics of $y$ and $\varphi$ can be safely neglected $F_{q}=0$.

The generalized friction force describing fluctuations of the phase corresponds to the current passing through the circuit impedance $Z_{e}$, that is

$$
F_{\varphi}=\frac{\hbar^{2}}{4 e^{2}} \int_{-\infty}^{t} d t^{\prime} d t^{\prime \prime} Z_{e}^{-1}\left(t^{\prime}, t^{\prime \prime}\right)\left[V_{b}-\dot{\varphi}\left(t^{\prime \prime}\right)\right] .
$$

With this, the equations of motion are

$$
\begin{gathered}
M \ddot{y}=-\frac{\partial U}{\partial y}-M \Gamma \dot{y} \\
0=-\frac{\partial U}{\partial q} \\
0=-\frac{\partial E_{j}}{\partial \varphi}+\frac{\hbar^{2}}{4 e^{2}} \int_{-\infty}^{t} d t^{\prime} d t^{\prime \prime} Z_{e}^{-1}\left(t^{\prime}, t^{\prime \prime}\right)\left[V_{b}-\dot{\varphi}\left(t^{\prime \prime}\right)\right]
\end{gathered}
$$

equivalent to those presented in the main text.

The equivalent Hamiltonian is

$$
\mathcal{H}=T(p)+U, \quad T(p)=p^{2} / 2 M,
$$

where $p$ is the momentum of the resonator $p=M \dot{y}$. There is no generalized momentum associated to $\varphi$ and $q$, since their inertial effects are not included in the Lagrangian.

Using the Hamiltonian, we obtain the same equations of motion using

$$
\begin{gathered}
\dot{p}=-\frac{\partial \mathcal{H}}{\partial y}+F_{y}, \\
0=-\frac{\partial \mathcal{H}}{\partial q}, \\
0=-\frac{\partial \mathcal{H}}{\partial \varphi}+F_{\varphi} .
\end{gathered}
$$

${ }^{1}$ A. N. Cleland, Foundations of Nanomechanics (Springer-Verlag, Berlin, 2003).

${ }^{2}$ K. L. Ekinci, X. M. H. Huang, and M. L. Roukes, Appl. Phys. Lett. 84, 4469 (2004)

${ }^{3}$ M. P. Blencowe and E. Buks, Phys. Rev. B 76, 014511 (2007).

${ }^{4}$ A. N. Cleland and M. L. Roukes, Nature (London) 392, 160 (1998).

${ }^{5}$ A. D. Armour, M. P. Blencowe, and K. C. Schwab, Phys. Rev. Lett. 88, 148301 (2002).

${ }^{6}$ T. L. Schmidt, K. Børkje, C. Bruder, and B. Trauzettel, Phys. Rev. Lett. 104, 177205 (2010).

${ }^{7}$ A. D. O'Connell, M. Hofheinz, M. Ansmann, R. C. Bialczak, M. Lenander, E. Lucero, M. Neeley, D. Sank, H. Wang, M. Weides, J. Wenner, J. M. Martinis, and A. N. Cleland, Nature (London) 464, 697 (2010).
${ }^{8}$ I. Wilson-Rae, N. Nooshi, W. Zwerger, and T. J. Kippenberg, Phys. Rev. Lett. 99, 093901 (2007); F. Marquardt, J. P. Chen, A. A. Clerk, and S. M. Girvin, ibid. 99, 093902 (2007).

${ }^{9}$ G. Sonne, M. E. Peña-Aza, L. Y. Gorelik, R. I. Shekhter, and M. Jonson, Phys. Rev. Lett. 104, 226802 (2010).

${ }^{10}$ M. D. LaHaye, J. Suh, P. M. Echternach, K. C. Schwab, and M. L. Roukes, Nature (London) 459, 960 (2009).

${ }^{11}$ H. G. Craighead, Science 290, 1532 (2000); M. Blencowe, Phys. Rep. 395, 159 (2004); K. L. Ekinci, and M. L. Roukes, Rev. Sci. Instrum. 76, 061101 (2005).

${ }^{12}$ J. Guckenheimer and P. Holmes, Nonlinear Oscillations, Dynamical Systems, and Bifurcations of Vector Fields (Springer-Verlag, New York, 1983). 
${ }^{13}$ V. Sazonova, Y. Yaish, H. Üstünel, D. Roundy, T. A. Arias, and P. L. McEuen, Nature (London) 431, 284 (2004).

${ }^{14}$ B. Witkamp, M. Poot, and H. S. J. van der Zant, Nano Lett. 6, 2904 (2006).

${ }^{15}$ G. A. Steele, A. K. Hüttel, B. Witkamp, M. Poot, H. B. Meerwaldt, L. P. Kouwenhoven, and H. S. J. van der Zant, Science 325, 1103 (2009).

${ }^{16}$ A. Krishnan, E. Dujardin, T. W. Ebbesen, P. N. Yianilos, and M. M. J. Treacy, Phys. Rev. B 58, 14013 (1998).

${ }^{17}$ A. Naik, O. Buu, M. D. LaHaye, A. D. Armour, A. A. Clerk, M. P. Blencowe, and K. C. Schwab, Nature (London) 443, 193 (2006).

${ }^{18}$ In more rigorous terms, $I$ is the moment of inertia per mass density per unit length of the cross section.

${ }^{19}$ L. D. Landau and E. M. Lifshitz, Theory of Elasticity (Pergamon, New York, 1986).

${ }^{20}$ S. Sapmaz, Y. M. Blanter, L. Gurevich, and H. S. J. van der Zant, Phys. Rev. B 67, 235414 (2003).
${ }^{21}$ G. Sonne, R. I. Shekhter, L. Y. Gorelik, S. I. Kulinich, and M. Jonson, Phys. Rev. B 78, 144501 (2008).

${ }^{22}$ In this case, the critical current is estimated from the product of the junction conductance and superconducting energy gap and can exceed the experimentally measured switching current by two orders of magnitude.

${ }^{23}$ K. K. Likharev, Dynamics of Josephson Junctions and Circuits (Gordon and Breach, New York, 1986).

${ }^{24}$ Y. V. Nazarov and Y. M. Blanter, Quantum Transport: Introduction to Nanoscience (Cambridge University Press, Cambridge, 2009).

${ }^{25}$ M. D. Fiske, Rev. Mod. Phys. 36, 221 (1964); D. D. Coon and M. D. Fiske, Phys. Rev. 138, A744 (1965).

${ }^{26}$ S. Shapiro, Phys. Rev. Lett. 11, 80 (1963).

${ }^{27}$ C. J. H. Keijzers, Ph.D. thesis, Delft University of Techonology, 2012.

${ }^{28}$ P. W. Anderson and A. H. Dayem, Phys. Rev. Lett. 13, 195 (1964). 